Applied Multivariate Research, Volume 13, No. 1, 2009, 43-76

\title{
THREE-WAY HIERARCHICAL CLASSES: A COMPARISON OF THE INDCLAS AND TUCKER3-HICLAS MODELS
}

\author{
Iwin Leenen \\ Universidad Complutense de Madrid \\ Eva Ceulemans \\ Katholieke Universiteit Leuven
}

\begin{abstract}
Hierarchical classes (HICLAS) models for multi-way multi-mode data constitute a unique family of classification models in that (a) they simultaneously induce a hierarchical classification (of the elements) of each mode and (b) they link the hierarchical classifications together by an association relation that yields a predicted (or reconstructed) value for each cell in the data array. For the case of three-way three-mode binary data, the most prominent HICLAS models include INDCLAS and Tucker3-HICLAS. In this paper, we compare the latter two models, introducing the underlying theory of both in substantive terms and showing how a Tucker3-HICLAS analysis may result in a simpler model than that yielded by INDCLAS, although the former is mathematically more complex than the latter (which it includes as a special case). We illustrate by two applications: a study on anger responses in frustrating situations and a case-study on emotions in interpersonal relations.
\end{abstract}

\section{INTRODUCTION}

Since De Boeck and Rosenberg's (1988) seminal paper on hierarchical classes, the HICLAS approach has expanded to a distinct family of classification models within the literature. The original model is a deterministic model for socalled two-way two-mode binary data. In this terminology (due to Tucker, 1964), the number of ways refer to the dimensionality of the data array, while the number of modes are the number of distinct sets these dimensions refer to. A well-known example of two-way one-mode data is a proximity (e.g., correlation) matrix, where rows and columns (two ways) of the matrix refer to the same set of entities/variables (one mode). For examples of two-way two-mode binary data, one may think of a child by item 0/1 matrix indicating success/failure of each child on each item, or object by attribute presence/absence data, person by choice object select/nonselect data, etc. Typically, deterministic models for two-way two-mode binary data (see, e.g., the models introduced by Coombs, 1964) include 


\section{APPLIED MULTIVARIATE RESEARCH}

an equation that assigns a predicted (or reconstructed model) $0 / 1$ value to each cell of the matrix. That is to say, these models account for the association relation between the two modes. In the same line, De Boeck and Rosenberg's model accounts for the association relation in a two-way two-mode binary array, but, as a unique feature, their model represents two other types of relations as well, namely the equivalence and hierarchy relations defined on the two modes of this array, which yield the typical two-sided hierarchical classification. Each of the three types of relations may be of substantive interest: For example, accounting for the association relation in person by object select/nonselect data may reveal the underlying choice mechanisms (Van Mechelen \& Van Damme, 1994); by representing the equivalence relations in patient by symptom presence/absence data, HICLAS may shed light on the diagnostic classification system of psychiatrists (Van Mechelen \& De Boeck, 1989); and the hierarchical relations are of key relevance in person perception (Gara, 1990; Gara \& Rosenberg, 1979), differential emotion psychology (Kuppens, Van Mechelen, Smits, De Boeck, \& Ceulemans, 2007), and knowledge space theory (Doignon \& Falmagne, 1999; Falmagne, Koppen, Villano, Doignon, \& Johannesen, 1990).

De Boeck and Rosenberg's (1988) original model has been extended in various directions: Variants have been proposed that differ in the way the association, equivalence, and hierarchy relations are represented (see, e.g., Van Mechelen, De Boeck, \& Rosenberg, 1995); generalizations have been developed that allow for a direct modeling of nonbinary data (Schepers \& Van Mechelen, 2006; Van Mechelen, Lombardi, \& Ceulemans, 2007); and other models replaced the deterministic framework by a probabilistic one (Leenen, Van Mechelen, Gelman, \& De Knop, 2008). Another extension of the original model comprises HICLAS models for $N$-way $N$-mode data (with $N>2$, in its general form described by Ceulemans and Van Mechelen, 2003), that is, data that can be represented in an $N$-dimensional array with the $N$ dimensions referring to $N$ different sets of elements. Substantively interesting HICLAS models for multi-way multi-mode data include the INDCLAS (Leenen, Van Mechelen, De Boeck, \& Rosenberg, 1999) and Tucker3-HICLAS model (Ceulemans, Van Mechelen, and Leenen, 2003; and variants of the latter, see Ceulemans and Van Mechelen, 2004, 2005), which are models for three-way three-mode binary data.

The present paper has a twofold aim: First, we want to provide an easy introduction to the INDCLAS and Tucker3-HICLAS models, focusing on substantive aspects. Therefore, we will recapitulate the theory behind these models giving special attention to the substantive meaning of the various aspects of the models. Furthermore, a number of tools that have been proposed to deal with the model selection problem - that is, the problem of selecting which INDCLAS or Tucker3-HICLAS model yields the best description of the data (best referring to

an optimal trade off between simplicity/parsimony and goodness of fit) - are discussed and illustrated, as this topic is particularly relevant for practical users. Second, we want to elaborate on the comparison of the INDCLAS and Tucker3HICLAS models, focusing on the results that can be expected when applying both models to the same data set. This will be illustrated by two applications. In the first application, it will be shown that a Tucker3-HICLAS model, which implies a generalization of the association rule in INDCLAS, may yield simpler hierarchical classifications. In the second application, we show how a Tucker3-HICLAS 


\section{THREE-WAY HICLAS}

model may yield a considerably better fit to the data as compared to an INDCLAS model of about the same complexity.

The paper is further organized as follows: In the next section, we give a precise definition of the association, equivalence, and hierarchy relations in threeway three-mode binary data and show how these relations are represented by the INDCLAS and Tucker3-HICLAS model, respectively. Subsequently, we discuss some of the more practical aspects (including model selection) when either model is fitted to a given data set. Thereafter, we discuss the two applications where we compare INDCLAS and Tucker3-HICLAS results. In the final section, we present some recent developments in HICLAS analysis and discuss their usefulness when applying the models discussed in this paper.

\section{The INDCLAS and Tucker3-HICLAS Model}

Consider an $I \times J \times K$ data array $\underline{\mathbf{X}}=\left(x_{i j k}\right)$ with entries $x_{i j k} \in\{0,1\} .{ }^{1}$ So as not to make the exposition unnecessarily abstract, we will illustrate the theoretical concepts introduced in this section by means of a hypothetical example. In particular, we will assume in this guiding example that four staff members in a health service-a clinical psychologist, a trainee, a general practitioner, and a psychiatrist - each interviewed (the same) six patients at intake — a drug addict, a paranoiac, a demented man, a compulsive cleaner, an anorexic girl, and a man suffering delirium - and, after the interview, judged the presence/absence of seven psychiatric symptoms - hallucinations, delusions, anxiety, depression, obsessive behavior, social isolation, and disorientation - in each of these patients. The data, which can be organized in a 4 (judges) $\times 6$ (patients) $\times 7$ (symptoms) data array $\underline{\mathbf{X}}$, with $x_{i j k}=1$ if judge $i$ considers symptom $k$ present in patient $j$ and $x_{i j k}=0$ otherwise, are shown in Table 1.

We now define three types of relations on such a three-way three-mode data array. Thereafter, we will show how the INDCLAS and Tucker3-HICLAS models account for these relations.

\section{Equivalence, Hierarchy, and Association in a Binary Three-Way Array}

Equivalence. Equivalence relations are defined on the set of elements of a given mode. As a result, for three-way three-mode data, three equivalence relations are considered. For the data in Table 1, an equivalence relation is defined on (a) the set of four judges, (b) the set of six patients, and (c) the set of seven symptoms. A pair of elements of a given mode is equivalent if and only if the submatrices associated with each of them are identical. In order to compare the submatrices associated with the respective elements, a representation of the data such as in Figure 1 may be helpful. The figure represents the data in three different ways, depending on which mode is used to segregate submatrices. To make the comparison easier, a value of one in a cell is represented by a cross, while cells with a zero value are left blank. Figure 1(a) shows the patient by symptom submatrix of each judge, from which it is easily checked that the clinical psychologist and the psychiatrist have identical submatrices and, hence, are equivalent for these data. In the same way, from Figure 1(b), which shows the patient by judge submatrix of each symptom, we can derive, for example, that hallucinations, 
APPLIED MULTIVARIATE RESEARCH

Table 1. Hypothetical Three-Way Three-Mode Binary Data Array

Clinical psychologist

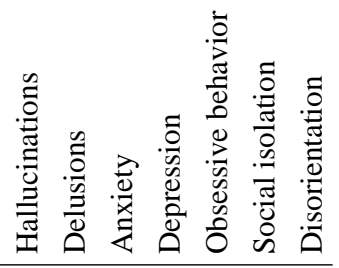

Drug addict

Paranoiac

Demented man

Compulsive cleaner $0 \begin{array}{lllllll}0 & 0 & 1 & 1 & 1 & 1 & 0\end{array}$

Anorexic girl

$\begin{array}{lllllll}0 & 0 & 1 & 1 & 1 & 1 & 0\end{array}$

$\begin{array}{llllllll}\text { Man with delirium } & 1 & 1 & 1 & 1 & 1 & 1 & 1\end{array}$
Trainee

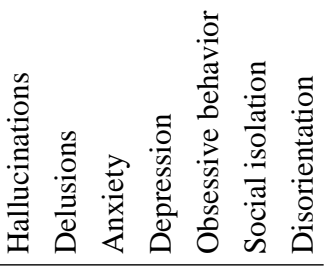

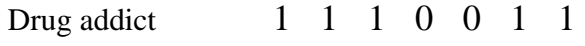

Paranoiac

$\begin{array}{lllllll}1 & 1 & 1 & 0 & 0 & 1 & 1\end{array}$

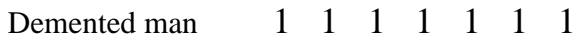

Compulsive cleaner $0 \begin{array}{lllllll}0 & 0 & 1 & 1 & 1 & 1 & 0\end{array}$

$\begin{array}{lllllllll}\text { Anorexic girl } & 0 & 0 & 1 & 1 & 1 & 1 & 0\end{array}$

$\begin{array}{llllllll}\text { Man with delirium } & 1 & 1 & 1 & 0 & 0 & 1 & 1\end{array}$
General practitioner

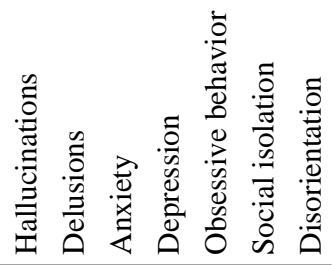

Drug addict

Paranoiac

Demented man

Compulsive cleaner

Anorexic girl

$\begin{array}{lllllll}0 & 0 & 0 & 0 & 0 & 0 & 0\end{array}$

$\begin{array}{llllllll}\text { Man with delirium } & 1 & 1 & 1 & 1 & 1 & 1 & 1\end{array}$
Psychiatrist

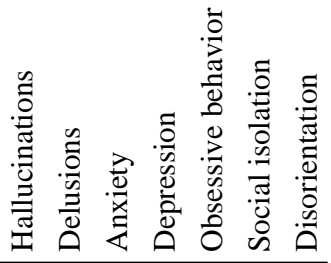

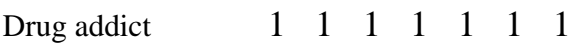

Paranoiac

$\begin{array}{lllllll}1 & 1 & 1 & 0 & 0 & 1 & 1\end{array}$

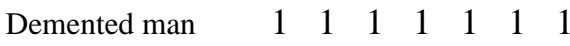

Compulsive cleaner $0 \begin{array}{lllllll}0 & 0 & 1 & 1 & 1 & 1 & 0\end{array}$

$\begin{array}{lllllllll}\text { Anorexic girl } & 0 & 0 & 1 & 1 & 1 & 1 & 0\end{array}$

$\begin{array}{llllllll}\text { Man with delirium } & 1 & 1 & 1 & 1 & 1 & 1 & 1\end{array}$ 
THREE-WAY HICLAS

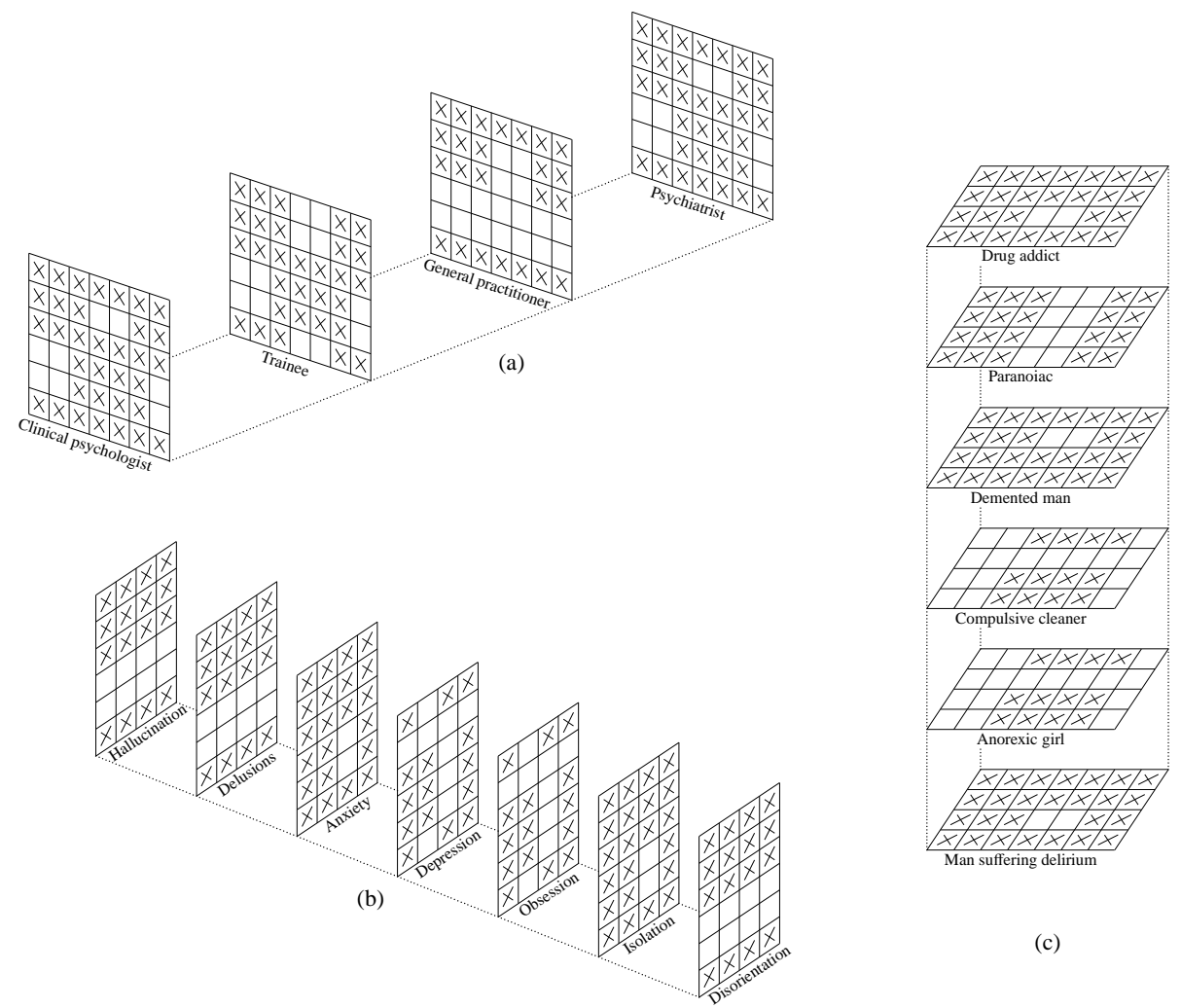

Figure 1. Three alternative representations of the data in Table 1: segregated (a) by judges, (b) by patients, and (c) by symptoms. Cells with a data value of 1 are filled with a cross; cells with a data value of 0 are left empty.

delusions, and disorientation are pairwise equivalent. Likewise, from Figure 1(c), which allows for a comparison of the judge by symptom submatrices of different patients, we learn, for example, that the compulsive cleaner and the anorexic girl are equivalent. The substantive interpretation of the equivalence relations follows from their definition: Equivalent judges assign the same symptoms when considering a particular patient; equivalent patients are assigned the same symptoms by each judge; and, for any judge, equivalent symptoms either co-occur or they are both absent in any patient. Note further that the equivalence relations induce a classification of the elements in the respective modes. For example, the equivalence relation on the symptoms induces the following three classes of equivalent symptoms: \{hallucinations, delusions, disorientation\}, \{anxiety, social isolation \}, \{depression, obsessive behavior\}.

Hierarchy. Apart from the equivalence relation, a hierarchy relation is defined on the set of elements of each mode. An element $i$ is hierarchically below an element $i^{\prime}$ if and only if the submatrix of $i$ is a proper subset of the submatrix of $i^{\prime}$. In tracing hierarchy relations, Figure 1 may again be helpful. From panel (a), we conclude, for example, that the trainee is hierarchically below the psychiatrist: If the trainee's matrix contains a cross, then the psychiatrist's matrix contains a 


\section{APPLIED MULTIVARIATE RESEARCH}

cross as well. This example shows that the hierarchy relation can alternatively be interpreted as an if-then type of relation (see also, Van Mechelen, Rosenberg, \& De Boeck, 1997): If the trainee judges a symptom to be present in a patient, then also the psychiatrist judges that symptom to be present in that patient. Likewise, from Figure 1(b), we can read that hallucinations are hierarchically below anxiety. That is, if a patient suffers from hallucinations (according to some judge) then (s)he suffers from anxiety as well (according to the same judge). Finally, Figure 1(c) shows that the paranoiac is hierarchically below the drug addict: If a symptom is believed to be present in the paranoiac, then it is believed to be present in the drug addict as well.

The hierarchy relation defined on the elements of a given mode directly produces a hierarchical organization of the classes induced by the equivalence relation. Such hierarchically organized classifications can be conveniently represented by a Hasse diagram (which is a graphical representation of a partially ordered set as a directed graph, the direction implicitly being upward, see Davey $\&$ Priestley, 2002). Figure 2 shows a Hasse diagram representing the hierarchical classification of the patients. Similar Hasse diagrams can be constructed for the judges and the symptoms as well. In such a representation, the boxes represent classes and the straight lines connecting the boxes indicate that the corresponding classes are hierarchically related. Equivalence and hierarchy relations are often alternatively called set-theoretical relations (as they are defined by comparing the sets of positive responses in the submatrices of different elements).

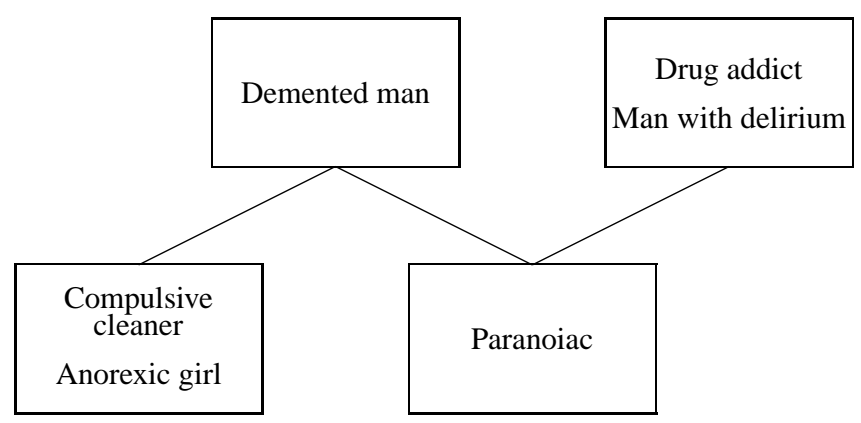

Figure 2. Hasse diagram representing the hierarchical classification of the patients as implied by the data in Table 1.

Association. The association relation links the three modes together. Loosely speaking, the association relation is "the" relation showed in the data array; more precisely, a judge $i$, a patient $j$, and a symptom $k$ are associated if and only if the corresponding cell in the data array has a value of one. Whereas the settheoretical relations are defined on each mode separately, giving rise to three equivalence and three hierarchy relations in a three-way three-mode array, there is only a single association relation, linking the three modes together. Analoguously to the way that the hierarchy relation on the elements implied a hierarchical organization of the classes, the association relation on the elements implies an association of the classes. Hence, the association relation can be said to link the three hierarchical classifications together. For example, from the data in Table 1, we read that the clinical psychologist, the drug addict, and hallucinations are 


\section{THREE-WAY HICLAS}

associated; as a result, the corresponding classes \{clinical psychologist, psychiatrist $\},\{$ hallucinations, delusions, disorientation $\}$, and \{drug addict, man suffering delirium $\}$ are associated as well.

\section{Representation of Equivalence, Hierarchy, and Association in INDCLAS}

Algebraically, an INDCLAS model for an $I \times J \times K$ binary array $\underline{\mathbf{X}}$ is a triple of binary matrices $(\mathbf{A}, \mathbf{B}, \mathbf{C})$ that satisfy a set of restrictions so as to represent the relations on $\underline{\mathbf{X}}$ that were introduced in the previous section. The dimensions of $\mathbf{A}$, $\mathbf{B}$, and $\mathbf{C}$ are $I \times R, J \times R$, and $K \times R$, respectively, where the integer $R$ is called the rank of the model. The $R$ columns of $\mathbf{A}, \mathbf{B}$, and $\mathbf{C}$ correspond to $R$ bundles and these matrices themselves are called bundle matrices. The bundles can be conceived of as latent features that are related in some way to the elements in the three modes. In some cases, the bundles can be given a substantive interpretation. As an example, consider Table 2, which shows an INDCLAS model of rank $R=3$ for the hypothetical data of Table $1 .^{2}$ For this example, we will interpret the bundles in terms of latent syndromes (for applications on similar, genuine data, see Leenen, Van Mechelen, De Boeck \& Rosenberg, 1999; Van Mechelen \& De Boeck, 1989), namely affective disorder, psychotic disorder, and substance abuse disorder, where the bundle matrix $\mathbf{A}$ indicates for each of the $(I=4)$ judges which of the $(R=3)$ latent bundles/syndromes (s)he takes into account, $\mathbf{B}$ indicates for each of the $(J=6)$ patients from which of the $(R=3)$ syndromes (s)he suffers, and $\mathbf{C}$ indicates for each of the $(K=7)$ symptoms of which of the $(R=3)$ syndromes it is characteristic. We see, for example, that the trainee takes into account the affective and psychotic disorders, but not the substance abuse disorder (possibly due to a lack of experience with the latter). Similarly, the paranoiac suffers from a psychotic disorder but not from affective and substance abuse disorders; and hallucinations are characteristic of psychotic and substance abuse disorders, but not of affective disorders.

One may note parallels between the models for hierarchical classes analysis and principal component analysis (PCA) and, in particular, the conceptual similarity between a bundle matrix in a HICLAS model on the one hand and a loading matrix or (component) score matrix in PCA on the other hand. Both a bundle and a loading/score matrix indicate for each of a number of observed entities (e.g., judges, patients, symptoms, persons, tests, items, etc.) the loading on or connection with a number of latent variables (with the obvious difference that loading and score matrices in PCA commonly have real-valued entries, while bundle matrices in HICLAS are binary).

We now discuss the restrictions that are imposed on the three INDCLAS bundle matrices so as to make them represent the abovementioned three types of relations.

Equivalence. If two elements of a given mode are equivalent (or, in other words, belong to the same class), then in the bundle matrix for that mode, the rows corresponding to these elements must be identical. For example, the clinical psychologist and the psychiatrist, which were found to be equivalent in the data of Table 1 (see, Figure 1(a)), have identical rows in the judge bundle matrix $\mathbf{A}$ (both are related to all latent bundles/syndromes). Likewise, the symptoms in the 
APPLIED MULTIVARIATE RESEARCH

Table 2. INDCLAS Model for the Hypothetical Data of Table 1.

\begin{tabular}{|c|c|c|c|}
\hline \multirow[b]{2}{*}{ Judge bundle matrix A } & \multicolumn{3}{|c|}{ Syndromes } \\
\hline & 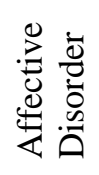 & 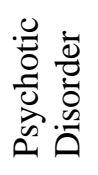 & 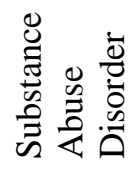 \\
\hline Clinical psychologist & 1 & 1 & 1 \\
\hline Trainee & 1 & 1 & 0 \\
\hline General practitioner & 0 & 1 & 1 \\
\hline Psychiatrist & 1 & 1 & 1 \\
\hline
\end{tabular}

Patient bundle matrix B

\begin{tabular}{llll}
\hline Drug addict & 0 & 1 & 1 \\
Paranoiac & 0 & 1 & 0 \\
Demented man & 1 & 1 & 0 \\
Compulsive cleaner & 1 & 0 & 0 \\
Anorexic girl & 1 & 0 & 0 \\
Man with delirium & 0 & 1 & 1 \\
\hline
\end{tabular}

Symptom bundle matrix $\mathrm{C}$

\begin{tabular}{llll}
\hline Hallucinations & 0 & 1 & 1 \\
Delusions & 0 & 1 & 1 \\
Anxiety & 1 & 1 & 1 \\
Depression & 1 & 0 & 1 \\
Obsessive behavior & 1 & 0 & 1 \\
Social isolation & 1 & 1 & 1 \\
Disorientation & 0 & 1 & 1 \\
\hline
\end{tabular}




\section{THREE-WAY HICLAS}

class \{hallucinations, delusions, and disorientation have identical rows in the symptom bundle matrix $\mathbf{C}$ (the three being characteristic of the psychotic as well as the substance abuse disorder but not of the affective disorder).

Hierarchy. Two elements of a given mode that are hierarchically related must have rows in the corresponding bundle matrix that are in a subset/superset relation. For example, we have already pointed out that, in the hierarchy relation defined on the judges, the trainee is below the psychiatrist; therefore, in the judge bundle matrix A, the trainee's row is a (proper) subset of the psychiatrist's row: The psychiatrist takes into account all the syndromes that the trainee takes into account (and more). Similarly, in the patient bundle matrix $\mathbf{B}$, as the paranoiac is below the drug addict in the patient hierarchy, the row of the former is a subset of the row of the latter: The paranoiac suffers from a subset of the syndromes that the drug addict suffers from.

Association. Saying that a hierarchical classes model represents the association relation means that the model includes a rule which allows to derive the binary value of a given data cell from the bundle matrices. For the INDCLAS model, this rule reads as follows: Three elements (from different modes) are associated if and only if there exist a latent bundle to which each of them is related. In the context of our guiding example: A judge $i$ indicates that patient $j$ suffers from symptom $k$ if and only if there exists a syndrome that (a) judge $i$ takes into account, (b) patient $j$ suffers from, and (c) symptom $k$ is characteristic of. Mathematically, this implies that the following equation holds for all $i, j$, and $k$ :

$$
x_{i j k}=\underset{r=1}{R} a_{i r} b_{j r} c_{k r},
$$

where $\oplus$ denotes the Boolean sum (i.e., with $1 \oplus 1=1$ ). One may check that, with model (A, B, C) in Table 2, Equation (1) holds for all cells of the data in Table 1. For example, the trainee judges the anorexic girl to suffer from depression (i.e., $x_{254}=1$ ) "because" there exists a syndrome (viz., affective disorder as represented by the first bundle) (a) that is taken into account by the trainee (i.e., $a_{21}=1$ ), (b) from which the patient suffers (i.e., $b_{51}=1$ ), and (c) of which depression is characteristic (i.e., $c_{41}=1$ ).

Leenen, Van Mechelen, De Boeck, and Rosenberg (1999) proposed a comprehensive graphical representation of the INDCLAS model from which (most of) the relations accounted for can be easily read. The graphical representation of the INDCLAS model in Table 2 is displayed in Figure 3. The upper part of the figure (above the circles in the center) shows the Hasse diagram with the hierarchical organization of the patients (in the same way as in Figure 2), while the bottom part (under the circles) shows, upside down, the Hasse diagram with the hierarchical organization of the symptoms. Each hierarchy has at the bottom $R=3$ bundle-specific classes (of which one or more may be empty). The elements in a bundle-specific class are exclusively related to one bundle (or syndrome) and, as a result, have exactly one value which equals one in their row of the bundle matrix. Whereas in the symptom hierarchy, all bundle-specific classes are empty (note that neither of the rows in bundle matrix $\mathbf{C}$ of Table 2 has exactly one value 


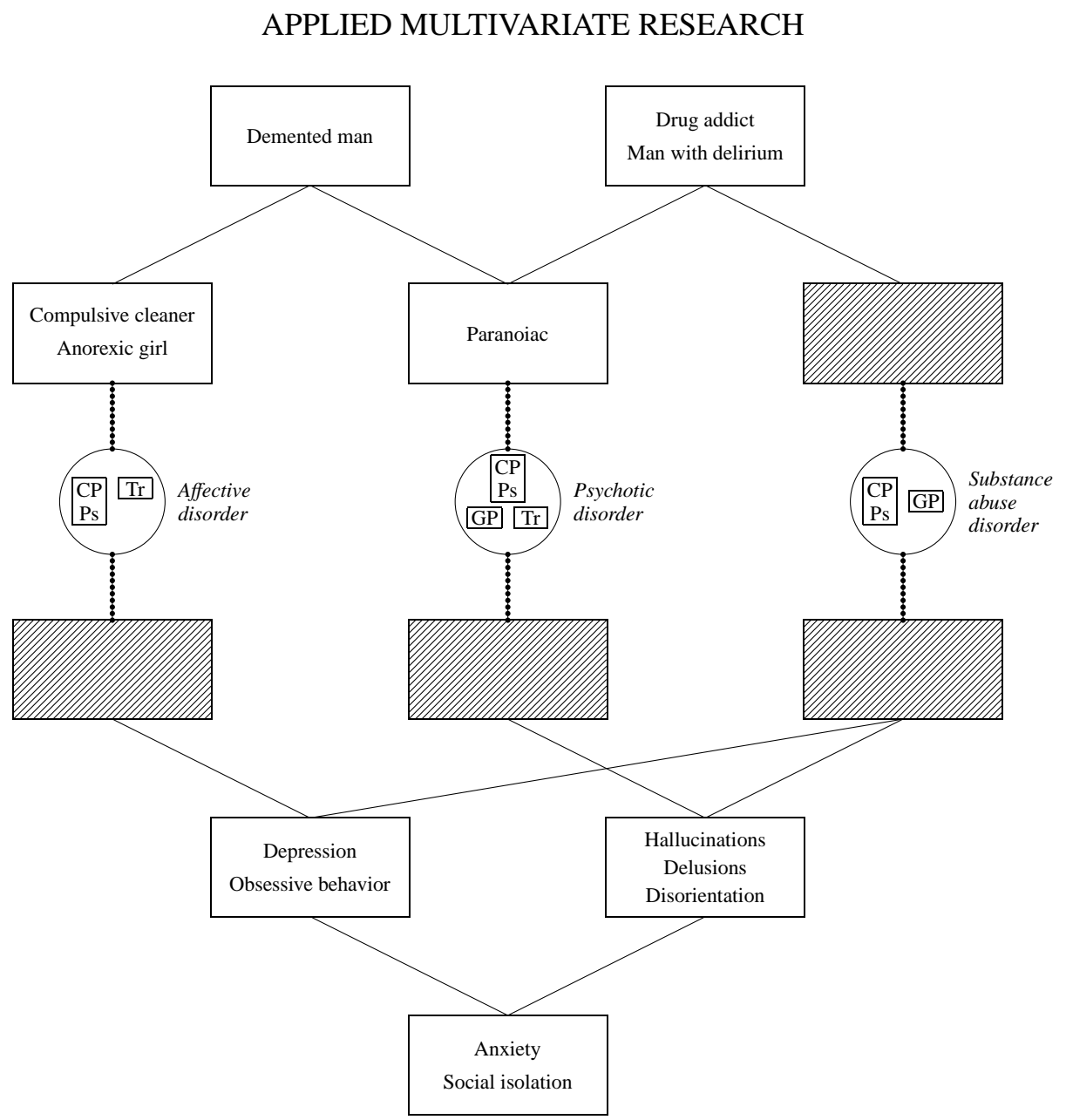

Figure 3. Graphical representation of the INDCLAS model of Table 2. CP, Tr, GP, and Ps stand for Clinical psychologist, Trainee, General practitioner, and Psychiatrist, respectively.

of one), the bundle-specific classes in the patient hierarchy are (i) \{compulsive cleaner, anorexic girl \}, (ii) \{paranoiac $\}$, and (iii) an empty class. The compulsive cleaner and the anorexic girl are exclusively related to the affective disorder, while the paranoiac suffers only from the psychotic disorder. In order to represent the association relation, the bundle-specific classes of the patient and symptom hierarchy are pairwise connected by circles, where each circle corresponds to a particular bundle/syndrome and contains the (classes of) judges who are related with that bundle (i.e., who take that syndrome into account). For example, the leftmost circle, corresponding to the affective disorder, contains the judge classes \{Clinical psychologist, Psychiatrist $\}$ and $\{$ Trainee $\}$, that is, the judges that have a one in the first position of their row in $\mathbf{A}$. As a consequence, the association relation is read as follows: If (and only if) there exists a (downward) path from patient $j$ to symptom $k$ that passes through a circle that contains judge $i$, then judge $i$, patient $j$, and symptom $k$ are associated. Thus, we can derive, for example, that the trainee indicates that the demented man suffers from depression, but that the general practitioner does not. 


\section{THREE-WAY HICLAS}

The graph does not show the hierarchical classification for the elements that are represented within the circles in the centre (in this case, the judges). ${ }^{3}$ Optionally, the hierarchical classification of these elements can be represented separately by means of a Hasse diagram, such as in Figure 4. Note that, like for the symptoms, the bundle-specific classes for the judges are all empty. These classes are nevertheless included so as to clarify with which syndromes the judges are related.

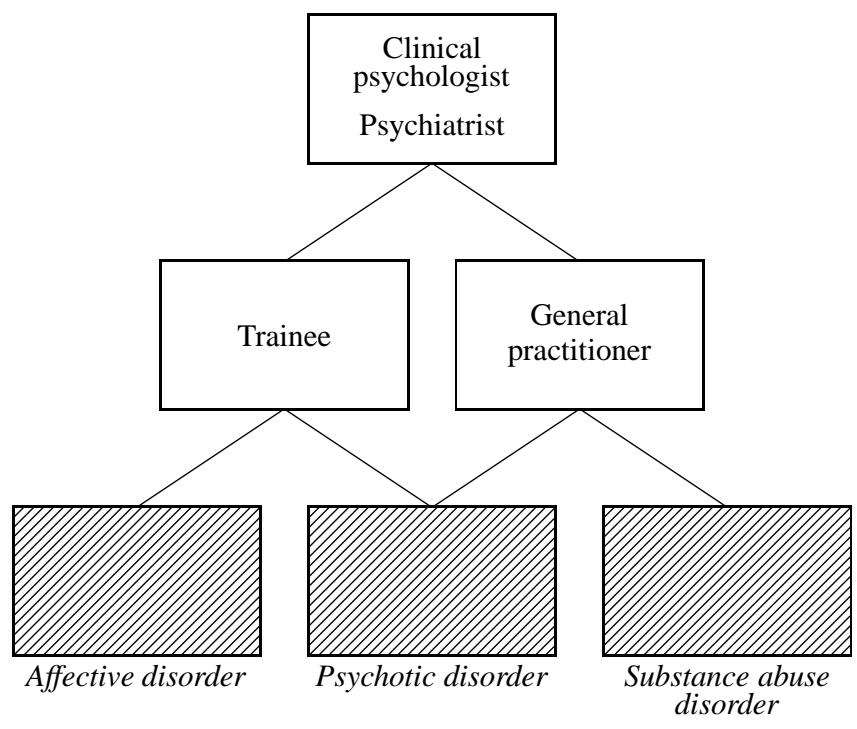

Figure 4. Graphical representation of the hierarchical classification of the judges in the INDCLAS model of Table 2.

The reader may wonder whether imposing the restriction in Equation (1) does not by itself imply that the bundle matrices correctly represent the set-theoretical relations. Or, in other words, is it possible that matrices $\mathbf{A}, \mathbf{B}$, and $\mathbf{C}$ correctly combine to $\underline{\mathbf{X}}$, while these matrices violate the restrictions with respect to equivalence and/or hierarchy? An example showing that this is possible indeed is given in Table 3. On the one hand, the data array $\underline{\mathbf{X}}$ can be perfectly reconstructed by Equation (1) from the bundle matrix $\mathbf{C}^{*}$ in this table and the bundle matrices $\mathbf{A}$ and $\mathbf{B}$ in Table 2. The set-theoretical relations, on the other hand, are not represented correctly. For example, the equivalent symptoms hallucinations and delusions do not have identical rows in $\mathbf{C}^{*}$; and disorientation is hierarchically below social isolation in $\underline{\mathbf{X}}$, while in $\mathbf{C}^{*}$ the row of the former is not a subset of the row of the latter. This triple $\left(\mathbf{A}, \mathbf{B}, \mathbf{C}^{*}\right)$ is characterized as a decomposition of $\mathrm{X}$ that is not set-theoretically consistent.

Representation of Equivalence, Hierarchy, and Association in Tucker3-HICLAS

In an INDCLAS model, the three modes share the same $R$ bundles; that is to say, the matrices $\mathbf{A}, \mathbf{B}$, and $\mathbf{C}$ have the same number of columns and the columns of these bundle matrices are in a one-to-one relationship (which is nicely illustrated in Figure 3 where the bundle-specific classes of patients and symptoms are 
Table 3. INDCLAS Symptom Bundle Matrix, which Together with Matrices A and B in Table 2 Correctly Represents the Association Relation, but Fails to Give a Correct Account of the Set-Theoretical Relations on the Symptoms as Implied by the Data in Table 1.

\begin{tabular}{|c|c|c|c|}
\hline \multirow[b]{2}{*}{ Symptom bundle matrix $C^{*}$} & \multicolumn{3}{|c|}{ Syndromes } \\
\hline & 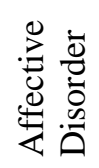 & 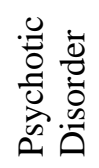 & 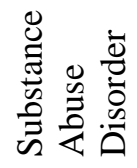 \\
\hline Hallucinations & 0 & 1 & 0 \\
\hline Delusions & 0 & 1 & 1 \\
\hline Anxiety & 1 & 1 & 0 \\
\hline Depression & 1 & 0 & 1 \\
\hline Obsessive behavior & 1 & 0 & 1 \\
\hline Social isolation & 1 & 1 & 0 \\
\hline Disorientation & 0 & 1 & 1 \\
\hline
\end{tabular}

connected through the bundle they have in common). The Hasse diagrams for judges and symptoms in Figures 3 and 4, however, show that, if only the hierarchical organizations of these modes are considered, a smaller number of bundles may be sufficient to represent them. This motivated the development of Tucker3HICLAS, which, like INDCLAS, implies a decomposition of a binary three-way three-mode array $\underline{\mathbf{X}}$ into bundle matrices $\mathbf{A}, \mathbf{B}$, and $\mathbf{C}$, but unlike INDCLAS, allows the bundle matrices to differ with respect to the number of columns. We write $I \times P, J \times Q$, and $K \times R$ for the dimensions of $\mathbf{A}, \mathbf{B}$, and $\mathbf{C}$, respectively. It follows that the rank is no longer characterized by a single number; rather, we will denote the rank of a Tucker3-HICLAS model as $(P, Q, R)$. Obviously, with the bundle matrices having a different number of columns and without further specification, it would be unclear how the bundles from different bundle matrices are connected (note that a one-to-one relationship like in INDCLAS is not generally possible anymore). Therefore, Tucker3-HICLAS adds a three-dimensional binary core array $\underline{\mathbf{G}}$, of dimension $P \times Q \times R$, which specifies $g_{p q r}=1$ if bundle $p$ of $\mathbf{A}$, bundle $q$ of $\mathbf{B}$, and bundle $r$ of $\mathbf{C}$ are connected, and $g_{p q r}=0$ otherwise.

By means of the Tucker3-HICLAS model of rank $(2,3,2)$ in Table 4 , we now show how the model represents the relations defined on the data array $\underline{\mathbf{X}}$ in Table 1 . 


\section{THREE-WAY HICLAS}

Equivalence and hierarchy. Tucker3-HICLAS imposes identical restrictions on $\mathbf{A}, \mathbf{B}$, and $\mathbf{C}$ as INDCLAS with respect to the representation of the set-theoretical relations. In the context of our guiding example, equivalent judges (respectively patients, symptoms) have identical rows in $\mathbf{A}$ (resp. B, C) and two judges (resp. patients, symptoms) that are hierarchically related have rows in $\mathbf{A}$ (resp. B, C) that are in a subset-superset relation. Note that, as to the set-theoretical relations, no restrictions are (directly) imposed on the core array $\underline{\mathbf{G}}$.

Association. In Tucker3-HICLAS, $\mathbf{A}, \mathbf{B}, \mathbf{C}$, and $\underline{\mathbf{G}}$ combine to $\underline{\mathbf{X}}$ by the following association rule:

$$
\begin{aligned}
& P Q R \\
& x_{i j k}=\bigoplus_{p=1} \underset{q=1}{\bigoplus} \underset{r=1}{\oplus} a_{i p} b_{j q} c_{k r} g_{p q r} .
\end{aligned}
$$

More substantively, within the context of the model in Table 4, this reads: A judge $i$ indicates that patient $j$ suffers from symptom $k$ if and only if (a) a judge bundle, say $p$, exists with which judge $i$ is related, (b) a patient bundle, say $q$, exists with which patient $j$ is related, (c) a symptom bundle, say $r$, exists with which symptom $k$ is related, and (d) judge bundle $p$, patient bundle $q$, and symptom bundle $r$ are connected in $\underline{\mathbf{G}}$. For example, the general practitioner judges that the drug addict suffers from depression (i.e., $x_{314}=1$ ) as (a) the general practitioner is related to judge bundle A-II (i.e., $a_{32}=1$ ), (b) the drug addict is related to patient bundle B-III (i.e., $b_{13}=1$ ), (c) depression is related to symptom bundle C-I (i.e., $c_{41}=1$ ), and (d) judge bundle A-II, patient bundle B-III, and symptom bundle C-I are connected in $\underline{\mathbf{G}}$ (i.e., $g_{231}=1$ ).

It is easily seen that the Tucker3-HICLAS model includes the INDCLAS model as a special case: A rank $R^{*}$ INDCLAS model $(\mathbf{A}, \mathbf{B}, \mathbf{C})$ is identical with a rank $(P, Q, R)$ Tucker3-HICLAS model $(\mathbf{A}, \mathbf{B}, \mathbf{C}, \underline{\mathbf{G}})$ with $P=Q=R=R^{*}$, and $g_{p q r}=1$ if $p=q=r$, and $g_{p q r}=0$ otherwise (i.e., $\underline{\mathbf{G}}$ is a unit superdiagonal array, Kiers, 2000).

A graphical representation of the Tucker3-HICLAS model in Table 4 is given in Figure 5. The Hasse diagram for the judges implied by the model is shown in Figure 6 . The relations of equivalence, hierarchy, and association are read from these graphs analoguously as from the INDCLAS graphs in Figures 3 and 4. Note that, on the one hand, the empty judge and symptom classes have disappeared in Tucker3-HICLAS, leaving simpler hierarchical classifications for these modes, while, on the other hand, the associations among the judges, patients, and symptoms (represented by the connections in the center of Figure 5) are slightly more complex than in the INDCLAS graph.

\section{Fitting a Three-Way HICLAS Model to Observed Data: Practical Considerations}

In going through the previous section, the reader might have raised the objection that it is particularly unlikely to find in a genuine three-way data array pairs of elements that are perfectly equivalent or that are hierarchically related without any exception. Indeed, in practical applications, the data almost never can be perfectly described by a simple HICLAS model, where with "simple" we mean "of low rank". (In this respect, remind that the rank poses an upper limit on the 
APPLIED MULTIVARIATE RESEARCH

Table 4. Tucker3-HICLAS Model for the Hypothetical Data of Table 1.

\begin{tabular}{|c|c|c|c|c|c|c|c|c|}
\hline \multicolumn{3}{|c|}{ Judge bundle matrix A } & \multicolumn{6}{|c|}{ Patient bundle matrix B } \\
\hline & A-1 & A-II & & & & B-I & B-II & B-III \\
\hline Clinical psychologist & 1 & 1 & \multicolumn{3}{|c|}{ Drug addict } & 0 & 1 & 1 \\
\hline Trainee & 1 & 0 & \multicolumn{3}{|c|}{ Paranoiac } & 0 & 1 & 0 \\
\hline General practitioner & 0 & 1 & \multicolumn{3}{|c|}{ Demented man } & 1 & 1 & 0 \\
\hline \multirow[t]{3}{*}{ Psychiatrist } & 1 & 1 & \multicolumn{3}{|c|}{ Compulsive cleaner } & 1 & 0 & 0 \\
\hline & & & Anore & xic $g$ & & 1 & 0 & 0 \\
\hline & & & Man v & ith & elirium & 0 & 1 & 1 \\
\hline \multicolumn{3}{|c|}{ Symptom bundle matrix $C$} & \multicolumn{6}{|c|}{ Core array $\underline{G}$} \\
\hline & C-I & C-II & A-I & & & A-II & & \\
\hline Hallucinations & 0 & 1 & & C-I & C-II & & C-I & $\mathrm{C}-\mathrm{II}$ \\
\hline Delusions & 0 & 1 & B-I & 1 & 0 & B-I & 0 & 0 \\
\hline Anxiety & 1 & 1 & B-II & 0 & 1 & B-II & 0 & 0 \\
\hline Depression & 1 & 0 & B-III & 0 & 0 & B-III & 1 & 0 \\
\hline Obsessive behavior & 1 & 0 & & & & & & \\
\hline Social isolation & 1 & 1 & & & & & & \\
\hline Disorientation & 0 & 1 & & & & & & \\
\hline
\end{tabular}

number of classes in a mode, namely $2^{R}$ if the bundle matrix for that mode has $R$ columns, as such, forcing a partition of its elements in $2^{R}$ hierarchically related classes). Although theoretically, for any data array, the rank can be increased up to a point that the HICLAS model represents the data perfectly (see, for a general treatment of existence in decomposition models for binary data, Leenen, Van Mechelen, \& De Boeck, 1999), in practical applications one usually allows for nonperfect HICLAS models but that are simple and easy to interpret. In the context of three-way HICLAS models, this means that the data array $\underline{\mathbf{X}}$ is approximated by an array $\underline{\hat{\mathbf{X}}}$, which can be perfectly reconstructed from a simple model, while an array $\underline{\mathbf{E}}$ accounts for the discrepancy between model and data:

$$
\underline{\mathbf{X}}=\underline{\hat{\mathbf{X}}}+\underline{\mathbf{E}}
$$




\section{THREE-WAY HICLAS}

where $\varepsilon_{i j k} \in\{-1,0,1\}$. The proposed INDCLAS and Tucker3-HICLAS algorithms (described in Leenen, Van Mechelen, De Boeck, \& Rosenberg, 1999, and Ceulemans et al., 2003, respectively) search, given a data array $\underline{\mathbf{X}}$ and a rank specified by the user, for a model [i.e., a triple $(\mathbf{A}, \mathbf{B}, \mathbf{C})$ and a quadruple $(\mathbf{A}, \mathbf{B}, \mathbf{C}, \underline{\mathbf{G}})$, respectively] that correctly represents the association, equivalence, and hierarchy relations in an array $\underline{\hat{\mathbf{x}}}$, while minimizing the errors in $\underline{\mathbf{E}}=\underline{\mathbf{X}}-\underline{\hat{\mathbf{X}}}$, that is, while minimizing the loss function:

$$
L(\underline{\hat{\mathbf{x}}})=\sum_{i=1}^{I} \sum_{j=1}^{J} \sum_{k=1}^{K}\left(x_{i j k}-\hat{x}_{i j k}\right)^{2}
$$

Since the values $x_{i j k}$ and $\hat{x}_{i j k}$ are binary, the least squares loss function in (2) is equivalent to a least absolute deviations loss function and returns the number of cells with different values for $\underline{\mathbf{X}}$ and $\underline{\hat{\mathbf{X}}}$. Such cells are called discrepancies. HICLAS algorithms aim at minimizing the number of discrepancies between a particular HICLAS model and observed data. Often, the value on loss function (2) is divided by the number of cells $(I \cdot J \cdot K)$, which yields the proportion of discrepancies. Note that the original minimization algorithms do not include restrictions on the bundle matrices and/or the reconstructed data array, which implies that the analysis is exploratory. Ceulemans, Van Mechelen, and Kuppens (2004) recently introduced some tools for fitting restricted HICLAS models, as such allowing for a confirmatory HICLAS analysis; this new development will be briefly discussed in the last section of this paper.

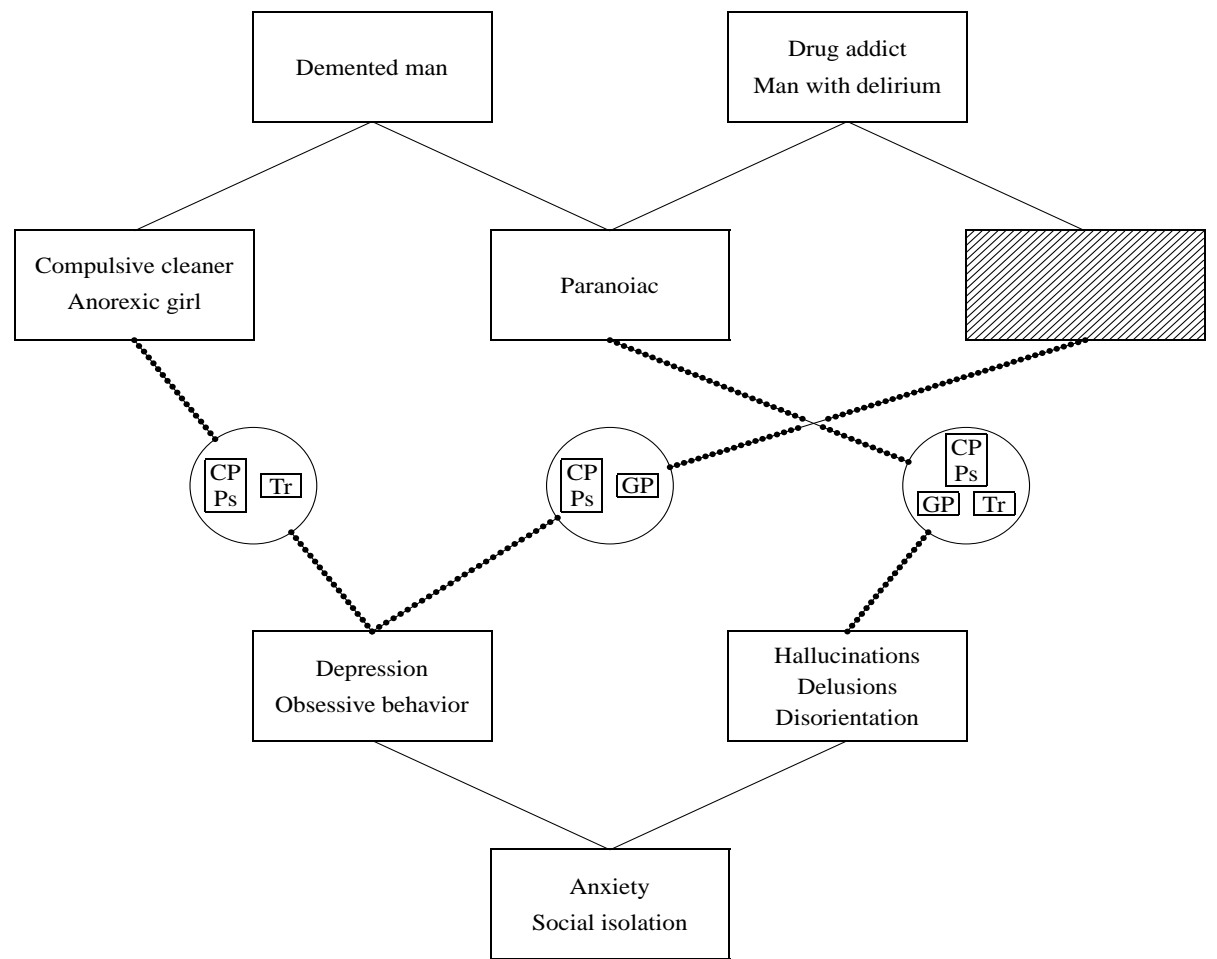

Figure 5. Graphical representation of the Tucker3-HICLAS model of Table 4. CP, Tr, GP, and Ps stand for Clinical psychologist, Trainee, General practitioner, and Psychiatrist, respectively. 


\section{APPLIED MULTIVARIATE RESEARCH}

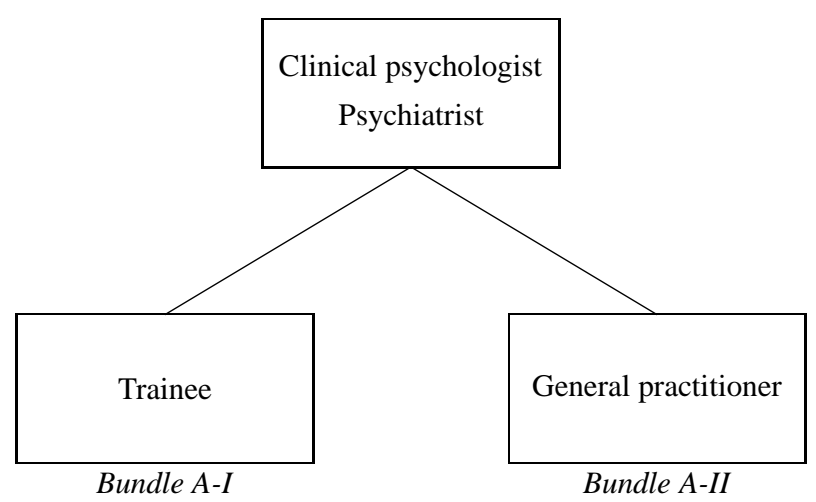

Figure 6. Graphical representation of the hierarchical classification of the judges in the Tucker3-HICLAS model of Table 4.

Although in some cases the rank of a HICLAS model follows from a theory about the data generation process, in most applications the basis for prespecifying the rank of the model (required as input to the fitting algorithms) is unclear. A common strategy in that case is fitting models with different ranks in successive runs of the algorithm and selecting a model that gives an optimal trade-off between simplicity and fit to the data (e.g., proportion of discrepancies). This idea has been concretized in various heuristics for rank selection, including "visual methods" applying an "elbow criterion" on the rank by goodness of fit plot (i.e., the scree test and its extensions such as the convex-hull approach, see Cattell, 1966; Ceulemans \& Van Mechelen, 2005) and "pseudo-statistical methods" (such as the pseudo-binomial test, Leenen \& Van Mechelen, 2001, and the pseudo-AIC strategy, Ceulemans \& Van Mechelen, 2005). In the application sections, we will illustrate some of these heuristics and briefly discuss their rationale (for the details, we refer to the original papers).

As a final note, we mention that an INDCLAS or Tucker3-HICLAS model may suffer from an indeterminacy, in some sense similar to the indeterminacy in principal component analysis. The problem in HICLAS is definitely much smaller than in PCA, because in most applications the HICLAS model can be shown to be unique. In contrast with PCA, however, the problem is much harder to delineate in the HICLAS case (where alternative solutions cannot be obtained through a "rotation" of the solution). Ceulemans \& Van Mechelen (2003) identified some sufficient conditions for uniqueness, one of them being that an INDCLAS model is unique if the bundle-specific classes are nonempty. A similar, slightly more complex proposition (including a condition for the core array $\underline{\mathbf{G}}$ as well) applies to the Tucker3-HICLAS model.

\section{Application 1: Behavioral Signatures in Situation-Response Profiles}

We now present the results of an INDCLAS and Tucker3-HICLAS analysis of questionnaire data previously reported by Vansteelandt and Van Mechelen (1998). These authors presented 54 first-year psychology students with 23 frustrating situations (see Table 5), and asked them to indicate for each situation which behaviors from a set of 15 anger responses (mentioned in the bottom half 
THREE-WAY HICLAS

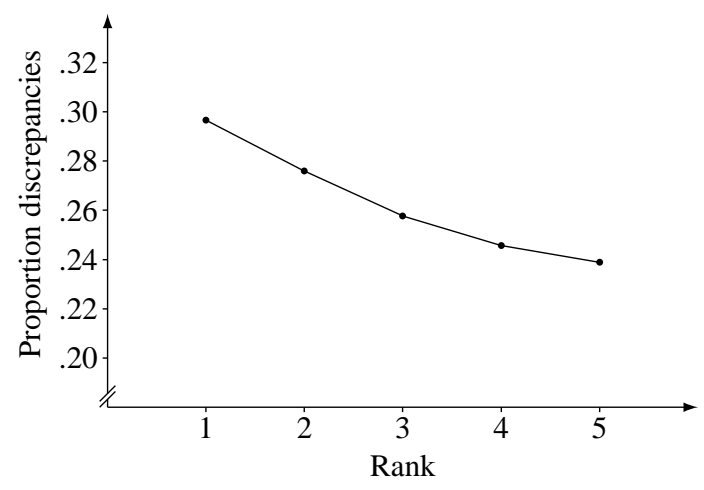

Figure 7. Plot of proportion of discrepancies by rank of five INDCLAS models for the situation-response data of Application 1.

of Figure 8) they would display, yielding a $54 \times 23 \times 15$ binary array $\underline{\mathbf{X}}$, with $x_{i j k}$ $=1$ if person $i$ displays behavior $k$ in situation $j$, and $x_{i j k}=0$ otherwise. The aim of the study was to construct from these data a triple typology model, that is, a model with (a) a typology (i.e., partition in classes) of persons, situations, and responses, (b) where the types in a typology are hierarchically organized, and (c) where the model would provide a characterization of the person types in terms of if(situation) - then(response) rules (called "behavioral signatures", Shoda, Mischel, \& Wright, 1994), as such linking the three typologies together. To this end, Vansteelandt and Van Mechelen analyzed the data array using the INDCLAS model. Here, we reanalyze the data with both the INDCLAS and the Tucker3-HICLAS algorithm. As will become clear in the discussion below, the solutions obtained for INDCLAS and Tucker3-HICLAS models of comparable complexity (i.e., of about the same rank) fit the data almost equally well. Therefore, we will concentrate on a comparison of the structural aspects of the INDCLAS and Tucker3-HICLAS models for these data.

\section{INDCLAS Analysis}

We successively applied the INDCLAS algorithm so as to find best fitting INDCLAS models in each of the ranks 1 to 5 and obtained models with proportions of discrepancies equal to $.297, .276, .258, .246$, and .239 , respectively. In order to select one of them for further discussion, we applied both visual and pseudo-statistical rank selection heuristics. Visual heuristics are based on an analysis of a fit by rank plot (see Figure 7) and, generally speaking, search for an "elbow" in such a plot (i.e., for a sharpest angle, Cattell, 1966). Pure visual inspection of the plot in Figure 7 would lead to a model in either rank 3 or 4 . A formalization of the "elbow" idea is Ceulemans et al.'s (2003; see also Leenen \& Van Mechelen, 2001) scree test, which calculates, for each rank $R$, the difference between the decrease in discrepancies when going from rank $R-1$ to $R$ and the decrease in discrepancies when going from rank $R$ to $R+1$, and selects the rank that maximizes this difference. A variant (see Ceulemans \& Van Mechelen, 2005) consists of calculating the ratio rather than the difference and selecting the rank that maximizes the ratio. ${ }^{4}$ If applied to the proportion of discrepancies for the five 
APPLIED MULTIVARIATE RESEARCH

Table 5. List of Frustrating Situations Used in the Situation-Response Questionaire of Application 1 With Key Words for Later Reference in Italic

1. You are talking to someone and he (she) does not answer you

2. You accidentally bang your shins against a park bench.

3. The grocery store closes just as you are about to enter.

4. Your instructor unfairly accuses you of cheating on an examination.

5. Someone has splashed mud over your new clothing.

6. Someone has lost an important book of yours.

7. Someone persistently contradicts you when you know you are right.

8. You have just found out that someone has told lies about you.

9. You are driving to a party and suddenly your car has a flat tire.

10. Someone makes an error and blames it on you.

11. You arrange to meet someone and he (she) does not show up.

12. You are reading a mystery and find that the last page of the book is missing.

13. You are trying to study and there is incessant noise.

14. You miss your train because the clerk has given you faulty information.

15. You are waiting at the bus stop and the bus fails to stop for you.

16. You are typing a term paper and your typewriter breaks.

17. You are in a restaurant and have been waiting a long time to be served.

18. You use your last 10c to call a friend and the operator disconnects you.

19. Someone has opened your personal mail.

20. You wake up early to get to a special 8 a.m. class and the instructor doesn't show up.

21. You are carrying a cup of coffee to the table and someone bumps into you.

22. You are very tired and have just gone to sleep, when you are awakened by the arrival of some friends.

23. Someone pushes ahead of you in a theaterticket line. 
THREE-WAY HICLAS

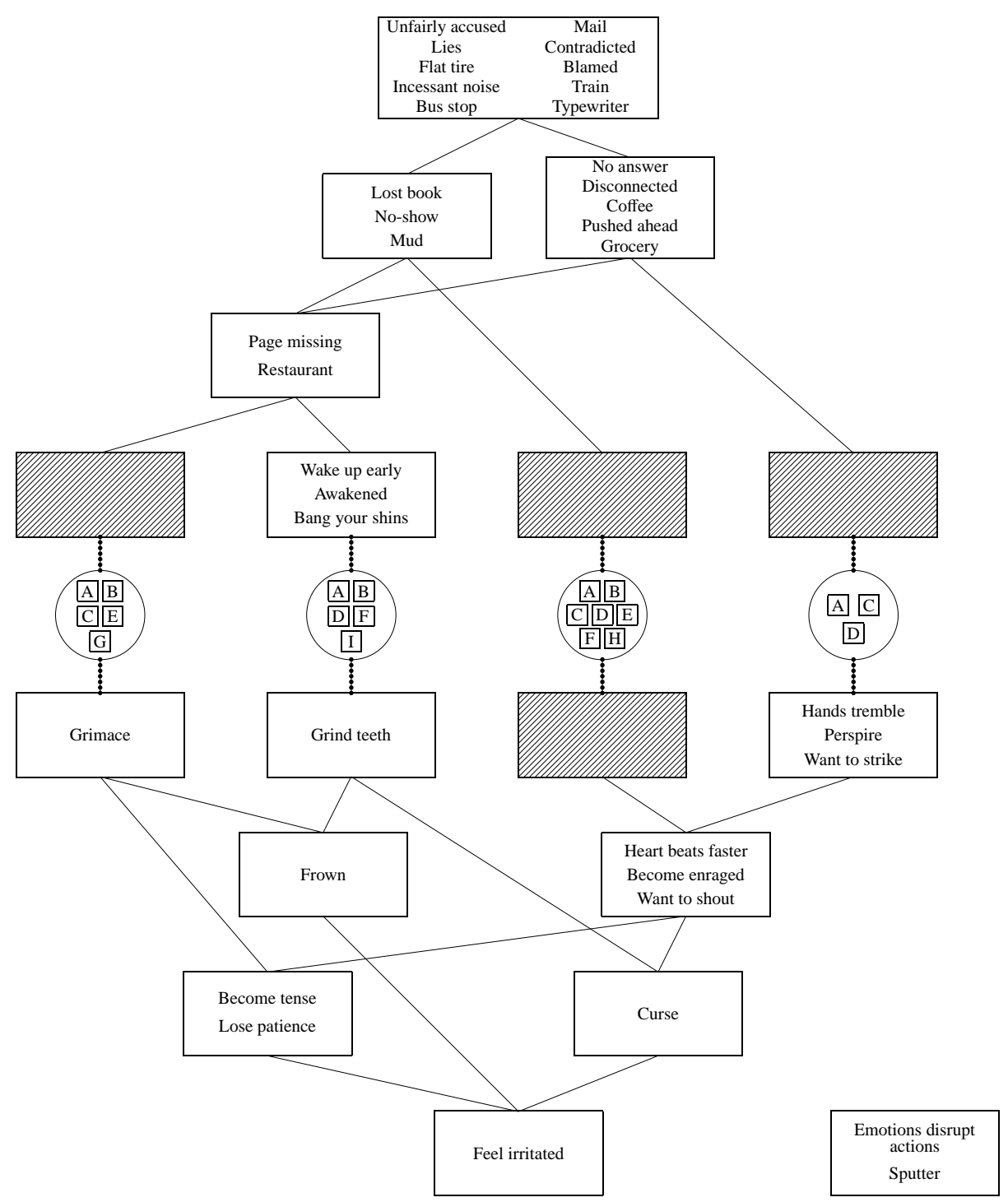

Figure 8. Graphical representation of the rank 4 INDCLAS model for the situation-response data of Application 1. Person classes in the circles are represented by capital letters. Person classes with a single element are omitted.

ranks, the difference-based scree test selects a rank 3 model, while the ratio-based variant selects a rank 4 model. In the proposed pseudo-statistical methods, the deterministic HICLAS model is considered within a probabilistic framework, assuming a stochastic process by which the cells of an underlying true binary array $\mathbf{T}$ may switch their values (either from 0 to 1 or vice versa) to give rise to the observed data $\underline{\mathbf{X}}$. The rationale of Leenen \& Van Mechelen's (2001) pseudobinomial heuristic is then to select the lowest rank $R$ for which the number of discrepancies in rank $R+1$ is not significantly lower than the number of discrep- 
ancies in rank $R$. Finally, the pseudo-AIC test proposed by Ceulemans \& Van Mechelen (2005) is based on Akaike's (1973; see also Bozdogan, 1987) information criterion (AIC) and selects the model in rank $R$, that minimizes

$$
-2 \log _{\mathrm{e}} L(\hat{\theta})+2 T
$$

where $L(\hat{\theta})$ is the likelihood of the model given the data within the presumed probabilistic framework and $T$ is the number of model parameters (i.e., the number of elements in the bundle matrices and, if applicable, the core array). When applying the pseudo-binomial and the pseudo-AIC test to the five fitted models, both point to the model in the highest rank (so, even a rank 6 model or higher may be considered most appropriate by these heuristics).

Considering, on the one hand, that most of the applied heuristics point to an INDCLAS model of rank 4 or higher, and, on the other hand, the difficulty in interpreting and representing higher-rank models (remind that in a rank 5 model, the Hasse diagram of a single mode may include up to 32 classes), we will retain the rank 4 model for further discussion. It is not unusual that the different rank selection heuristics yield inconsistent results. Anyhow, a decision on the rank of the model is preferably based not only on the outcome of one or more numerical procedures, but also on substantive considerations (interpretability). As an aside, we mention that Vansteelandt and Van Mechelen (1998) selected a rank 3 model based on an elbow "discovered" by visual inspection and substantive interpretability of the resulting solution.

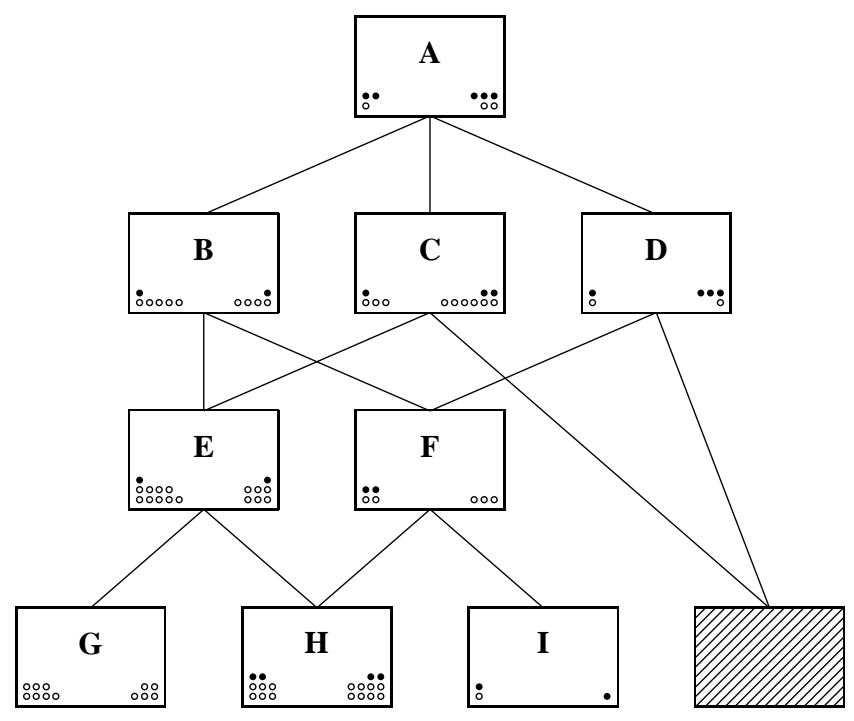

Figure 9. Graphical representation of the person typology in the INDCLAS and Tucker3-HICLAS models of Application 1. The number of white and black dots in the bottom left [right] corner of each box indicates how many women and men, respectively, belongs to that class in the INDCLAS [Tucker3-HICLAS] model. For simplicity's sake, the null class (with four females for each model) and single-person classes (four in INDCLAS and two in Tucker3-HICLAS) are omitted. 


\section{THREE-WAY HICLAS}

The rank 4 model is shown in Figures 8 and 9. From these figures, we see how the INDCLAS model meets the objectives aimed for by the original authors. A classification of the elements in each of the three modes is obtained, yielding a triple typology of persons, situations, and responses, where each of the typologies has an internal hierarchical structure. From Figure 8, we see that the 23 situations group into five types, where the type (class) at the top of the hierarchy include the situations that provoke the widest range of anger reactions. Indeed, from the definition of hierarchy it follows that if some situation provokes a particular anger reaction in a person, any situation that is hierarchically higher provokes that reaction in this person as well. The five situation classes almost form a complete order, with only at the third level two classes that are not hierarchically related. A complete order is indicative of a quantitative dimension, while classes that are not hierarchically related differ qualitatively (Gati \& Tversky, 1982). The qualitative difference between the classes at level three might relate to the one at the right side (with situations "no answer", "disconnected", etc.) covering situations implying incidental harm, while the one at the left side (with the situations "lost book", "no-show", etc.) seems to include situations where the harm is a consequence of neglect with the cause not being present in the situation.

The 15 responses are spread across 9 classes, with, as a consequence, several classes containing only a single response. Nevertheless, a substantive meaning can be given based on the bundles shared by the same classes. That way, classes which load on the fourth bundle (i.e., classes at the right side of the hierarchy in Figure 8) are more related to fysiological and more automatic responses, while bundles I and II seem indicative of more voluntary responses. As an aside, note the response class at the right bottom of Figure 8, which is not related to any other class. This class is called the null class and contains the elements that are not related to any bundle (i.e., the element's row in the bundle matrix only contains zero's, hence its name); this implies that (discrepancies aside) the responses in this class are never displayed by any person in any situation. In a graphical representation of a HICLAS model, the null class is put aside or (most often) not displayed at all. When going up in the hierarchy of the responses, the classes include more general responses. For example, feeling irritated is common to all situations (in the sense that it is the "first" response displayed in any situation, i.e., if a situation provokes anger responses from a person, these responses necessarily include feeling irritated), while perspiring, trembling, and wanting to strike are specific to the most frustrating situations only.

The person typology has persons distributed across 14 classes (including the null class, which contains persons who, according to the model, never display any anger response). So as to avoid cluttering up the graphical representations, we omitted in Figures 8 and 9 four single-person classes. The person types higher up in the hierarchy generally display more anger responses. For an interpretation of the person typology, one may look for a relation between characteristics of the person and the types they belong to. As a simple example, we will examine the distribution of both sexes across the classes. In Figure 9, we have indicated the number of women (white dots) and men (black dots) at the left bottom of each box. At simple inspection, it seems that men are relatively higher up in the hierarchy than women, which would mean that men are more prone to displaying anger responses than women. This impression is confirmed by a significant 


\section{APPLIED MULTIVARIATE RESEARCH}

polychoric correlation between a person's level in the hierarchy and his/her sex ( $r$ $=.39$, with 95\%-confidence interval $[.06, .73]$ ). More advanced (and far more interesting) comparisons between different types in the person typology may look for relations with a person's subjective appraisals, evaluative affective judgements, and/or his action tendencies (see Mischel \& Shoda, 1995; Vansteelandt \& Van Mechelen, 1998).

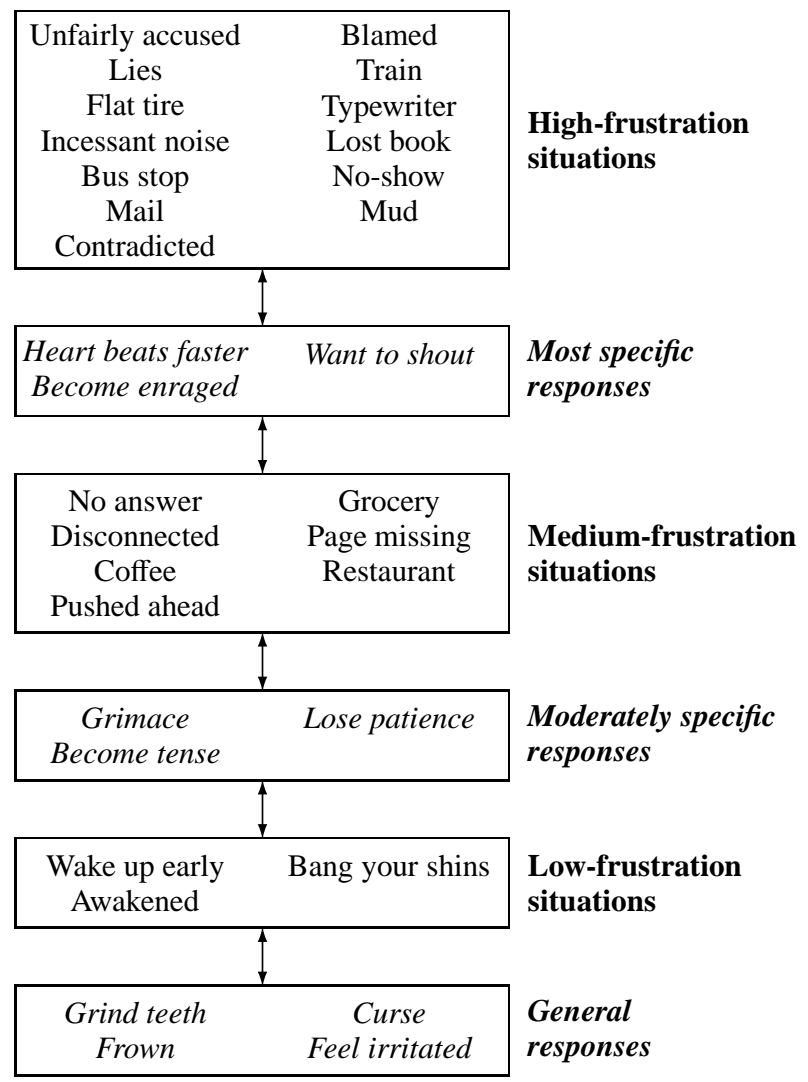

Figure 10. The total order of situation types (roman font) and response types (italic font) for Person type B in the INDCLAS model for the data in Application 1.

The INDCLAS association rule connects the typologies of situations, responses, and persons together. This allows for deriving, for each person type, a set of if-then rules that characterize them in terms of the responses they display in different situations. As an example, consider person type B. From Figure 8, it follows that these persons differentiate between (i.e., display distinct behaviours in) three groups of situations, which may be labeled high-, medium-, and low-frustration. Indeed, as persons of person type B are not related to the fourth bundle, they do not differentiate between situations in the top class (with "unfairly accused", "lies", etc.) and in the level-three class with "lost book", "no-show", and "mud", and, as a result, all these situations are regrouped into a single class 


\section{THREE-WAY HICLAS}

when considering person type B separately. For the same reason, also the class with, amongst others, "no answer", "disconnected" and the class with "page missing" and "restaurant" are joined together for person type B. Along the same lines, the behaviors for these persons are regrouped in three response classes, from most general to most specific. Figure 10 shows how these situation and response types are related: For a given situation type, persons of person type B display all responses below the situation type. Hence, if such a person is in a lowfrustration situation, then (s)he will display the general anger responses such as grind teeth, curse, and so on; if (s)he is in a medium-frustration situation, then (s)he will additionally grimace, become tense, and lose patience; finally, if (s)he finds him-/herself in a high-frustration situation, then (s)he will display the previous responses and the specific, fysiological/automatic responses. Such a set of if(situation)-then(response) rules for a given person (type) has been called his/her "behavioral signature" (Shoda et al., 1994), as it describes his/her particular way of behaving across situations of a given domain.

\section{Tucker3-HICLAS Analysis and Comparison With INDCLAS}

We applied the Tucker3-HICLAS algorithm to the same data to obtain models for all combinations of ranks $(P, Q, R)$ with $P, Q, R \in\{1, \ldots, 5\}$. Obviously, here the rank selection problem is considerably larger than in INDCLAS although, as pointed out by Ceulemans et al. (2003), some models are overparametrized and need not be evaluated (with $P, Q, R \in\{1, \ldots, 5\}$, only 74 of the $5^{3}=125$ models need to be evaluated). We will apply the convex-hull based approach proposed by Ceulemans and Van Mechelen (2005) for model selection, which is an extension of the scree test and is based on a plot of the proportion of discrepancies by the sum of the number of bundles $P+Q+R$. In a first step, this method retains all models that are on the lower boundary of the convex hull for the set of points. As shown in Figure 11, this procedure applied to the 74 models for the current data set retains seven models. (Remind that the convex hull of a set $S$ of points is the smallest convex set containing $S$; in this case, the set of points included in the polygon in Figure 11). A further selection of the retained models can then be

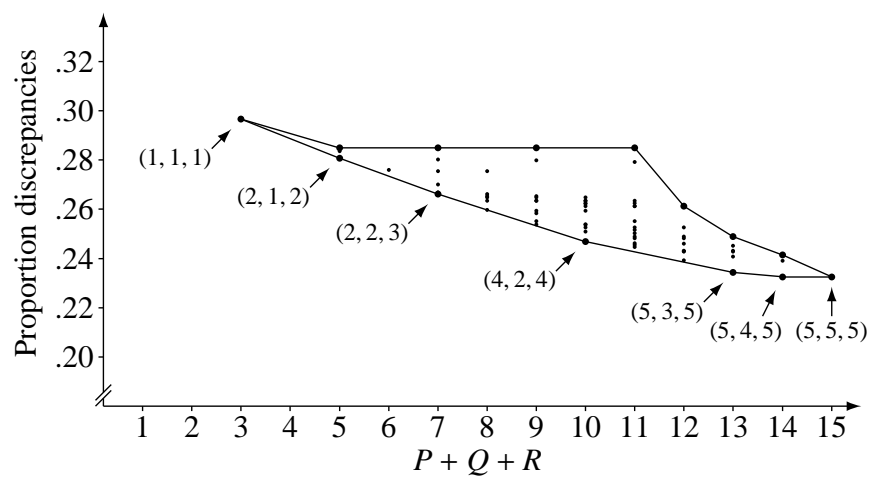

Figure 11. Plot of proportion of discrepancies by sum of the number of bundles $(P+Q+R)$ for the Tucker3-HICLAS models fitted to the situationresponse data of Application 1, with the ranks of the models on the lower boundary of the convex hull. 
obtained by applying a scree test similar to the one we applied for model selection in INDCLAS. With this approach, model $(5,3,5)$ and $(4,2,4)$ are almost indifferent (with a slight preference for the more complex model). For simplicity's sake, we will retain the $(4,2,4)$ model for further discussion. Note that the fit of the retained model (with a proportion of discrepancies equal to .247) is about the same as that of the INDCLAS model discussed in the previous section.

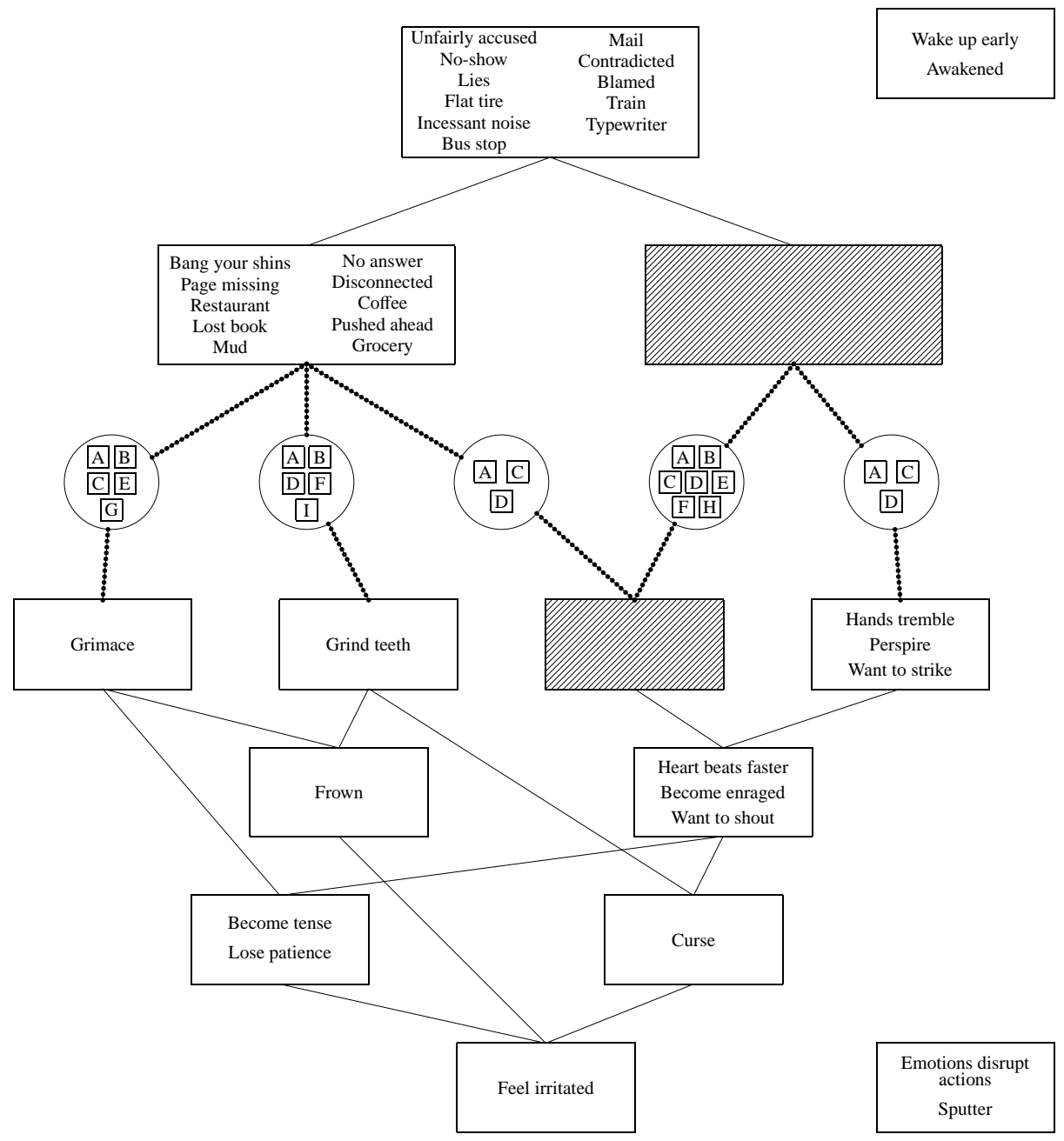

Figure 12. Graphical representation of the rank $(4,2,4)$ Tucker3-HICLAS model for the situation-response data of Application 1. Person classes in the circles are represented by capital letters.

The graphical representation of the $(4,2,4)$ model is shown in Figure 12 (with the hierarchical classification of the persons in Figure 9). Interestingly, the typology for the responses is identical to that in the INDCLAS model (Figure 8) and also the person typology, although not exactly identical, strongly resembles the typology in INDCLAS. This is not necessarily the case, even though both the 


\section{THREE-WAY HICLAS}

response and the person typology are based on four underlying bundles in the Tucker3-HICLAS as well as the INDCLAS model. With respect to the situations, the Tucker3-HICLAS model presents a strong simplification as compared to INDCLAS, with three situation classes that are totally ordered for all persons. As the model indicates that the two situations "wake up early" and "awakened" in the null class do not provoke any frustration in any of the persons and, therefore, can be left aside when interpreting the model, the situations that possibly provoke any anger reaction can be classified in a low-frustration type ("bang your shins", "no answer", etc.) and a high-frustration type ("unfairly accused", "mail", etc.).

The simplification in the situation typology is compensated for by a slightly more complex association rule, which no longer implies a one-to-one relation among the bundle-specific classes of the three typologies like in the INDCLAS model. However, the behavioral signatures of the respective person types are as easy to read from the graphical representation as in INDCLAS. For example, one might examine the if(situation) - then(response) rules of Person type B and find that situation and response classes can be totally ordered, similar to Figure 10. Thanks to the simplification in the situation typology, the retained Tucker3HICLAS model then guarantees that situation and response types are totally ordered for each person type. With the response and person typology as well as the goodness of fit being practically identical for the INDCLAS and Tucker3HICLAS model for these data, the simplification in the situation typology (and the resulting total order of situation and response types for each person) can be considered a net gain of the latter model.

\section{Application 2: A Single-Case Study on Interpersonal Emotions}

In our second application, we apply the INDCLAS and Tucker3-HICLAS models to three-way three-mode data obtained from a single (21-year old, female) individual (Geluykens, 2000), who was asked every 15 days over a five-month period (i.e., at 10 occasions) which of 40 emotions she experienced at that moment towards six target persons (mother, father, partner, and three relations that she characterized as unstable). This yielded a $10 \times 6 \times 40$ (occasion by target person by emotion) data array $\underline{\mathbf{X}}$, with $x_{i j k}=1$ if, at occasion $i$, this young woman indicated that she experienced emotion $k$ towards target person $j$, and $x_{i j k}=0$ otherwise.

We fitted INDCLAS models with rank $R$ ranging from 1 to 5 and Tucker3HICLAS models with rank $(P, Q, R)$ satisfying $P, Q, R \in\{1, \ldots, 5\}$ to these data. The dots in Figure 13 represent the proportions of discrepancies associated with the fitted Tucker3-HICLAS models in function of the sum of the number of bundles $P+Q+R$. For the sake of a first (rather crude) comparison, we have also represented, by means of squares, the proportions of discrepancies of the five fitted INDCLAS models, using $R+R+R$ as the corresponding complexity measure on the abscissa. It should however be noted that comparing the complexity of INDCLAS and Tucker3-HICLAS this way implies a disadvantage for the former model; as explained previously, a rank $R$ INDCLAS model is a restricted rank $(R, R, R)$ Tucker3-HICLAS model, yet the complexity measure used on the abscissa in Figure 13 returns equal values for both these models. Therefore, we 
first derive the convex hull for the set of Tucker3-HICLAS models and then we compare the Tucker3-HICLAS models that are located on the lower boundary of the convex hull with the INDCLAS models in rank 1 to 5 by their pseudo-AIC value, as defined in Equation (3). The pseudo-AIC test, as it accounts for the additional number of parameters in the core array of the Tucker3-HICLAS model, implies a fair comparison of the latter model with the more restricted INDCLAS model.

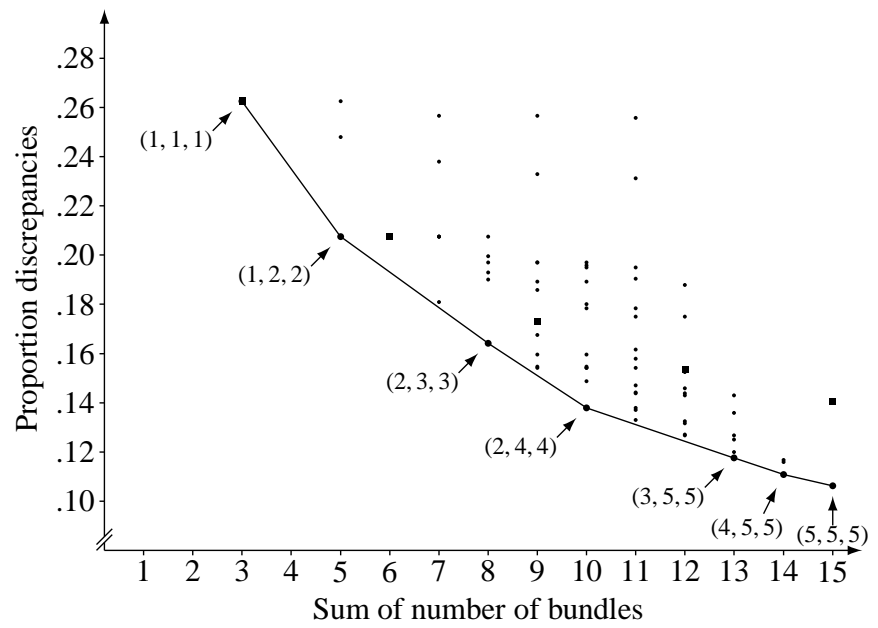

Figure 13. Plot of the proportion of discrepancies by the sum of the number of bundles for the INDCLAS (squares) and Tucker3-HICLAS (dots) models fitted to the interpersonal emotion data of Application 2, with the ranks of the (Tucker3-HICLAS) models on the lower boundary of the convex hull.

As shown in Figure 13, the lower bound of the convex hull contains seven Tucker3-HICLAS models. As an aside, one may note that applying a scree test (similar to the procedure applied in the previous application) would select the rank $(2,4,4)$ model from this set. Including the five INDCLAS models to the set of seven Tucker3-HICLAS models on the convex hull yields the twelve models shown in Table 6. From this table, we further read that the rank $(2,4,4)$ Tucker3HICLAS model has the lowest pseudo-IAC value. Moreover, we see that the INDCLAS models have considerably worse fit than their Tucker3-HICLAS counterparts: The selected rank $(2,4,4)$ Tucker3-HICLAS model decreases the number of discrepancies in the INDCLAS rank 4 model with about $10 \%$ (and fits even slightly better than the INDCLAS rank 5 model). Here, with the rank $(2,4,4)$ Tucker3-HICLAS and the rank 4 INDCLAS model having about the same degree of parsimony, the significant improvement in goodness of fit can be considered a net gain of the former model. Given that Tucker3-HICLAS models for these data clearly fit better than the INDCLAS models, we limit further (substantive) discussion to the retained $(2,4,4)$ Tucker3-HICLAS model.

Figure 14 displays the graphical representation of the selected model. We first discuss the hierarchical classification obtained for each of the three modes. The circles in the center of the figure show how the ten time points are grouped in three classes. Interestingly, each class contains a set of consecutive time points, as 
THREE-WAY HICLAS

Table 6. Discrepancies, Number of Parameters, and Pseudo-AIC Values for the INDCLAS models and a Selected Set of Tucker3-HICLAS Models Fitted to the Interpersonal Emotion Data of Application 2.

\begin{tabular}{lccccc}
\hline Model type & \multicolumn{5}{c}{$\begin{array}{c}\text { Number of } \\
\text { Rank discrepancies }\end{array}$} \\
\hline Iiscrepancies & $\begin{array}{c}\text { Number of } \\
\text { parameters }\end{array}$ & $\begin{array}{c}\text { Pseudo-IAC } \\
\text { value }\end{array}$ \\
\hline INDCLAS & 1 & 630 & .263 & 56 & 2875.15 \\
Tucker3-HICLAS & $(1,1,1)$ & 630 & .263 & 57 & 2877.15 \\
Tucker3-HICLAS & $(1,2,2)$ & 498 & .208 & 106 & 2663.00 \\
INDCLAS & 2 & 498 & .208 & 112 & 2675.00 \\
INDCLAS & 3 & 415 & .173 & 168 & 2546.31 \\
Tucker3-HICLAS & $(2,3,3)$ & 394 & .164 & 176 & 2495.27 \\
INDCLAS & 4 & 368 & .153 & 224 & 2504.55 \\
Tucker3-HICLAS & $(2,4,4)$ & 331 & .138 & 236 & 2397.58 \\
INDCLAS & 5 & 337 & .140 & 280 & 2507.45 \\
Tucker3-HICLAS & $(3,5,5)$ & 282 & .118 & 335 & 2407.19 \\
Tucker3-HICLAS & $(4,5,5)$ & 266 & .111 & 370 & 2411.62 \\
Tucker3-HICLAS & $(5,5,5)$ & 255 & .106 & 405 & 2435.29 \\
\hline
\end{tabular}

such subdividing the complete five-month period into three phases (where the three phases comprise occasions 1 to 6 [T1-T6], occasions 7 to 9 [T7-T9], and the single last occasion [T10], respectively). Note that the grouping in consecutive time points does not follow from a restriction in the model, but is important from a substantive point of view. The hierarchical relations among the three phases, which are not directly shown in Figure 14, are of less substantive interest and will not be discussed further.

With a grouping into five classes, we can hardly speak of a classification of the six target persons. We observe that the partner and one of the unstable relations classify together, which implies that the participant experienced the same emotions towards these two persons across the five-month period. Furthermore, we observe that the emotions this woman experienced towards her father are a combination of the emotions towards her partner (and Unstable relation A) on the one hand and her mother on the other hand. Surely, it is too far-fetched to adhere psycho-analytical interpretations about the father figure of this woman on this result only, but if such results were obtained from an analysis of a patient's data in a clinical setting, they may yield hypotheses to a psychiatrist, which then can be further investigated in one or more therapy sessions.

In the hierarchical classification of the 40 emotions, we see that all positive emotions are related to the first bundle (at the left side in Figure 14), with higherup in the hierarchy more general positive emotions (e.g., friendly) and in the 


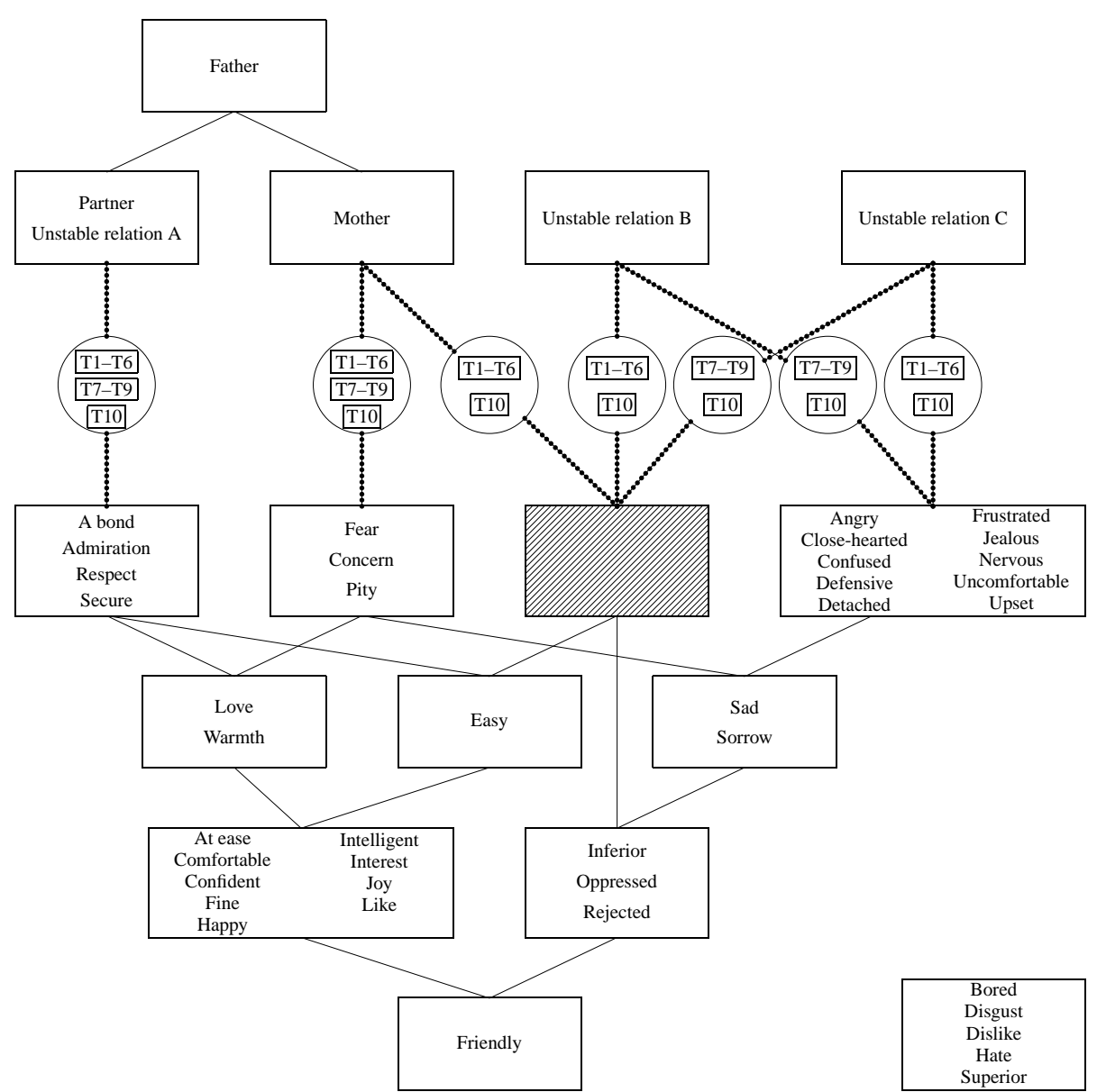

Figure 14. Graphical representation of the rank $(2,4,4)$ Tucker3-HICLAS model for the interpersonal emotion data of Application 2.

bottom class strong positive feelings (e.g., admiration). Emotions that are not related to the first bundle are either negative (particularly those related to the fourth bundle at the right side of the graph) or indicate ambivalence (such as concern and pity, which are exclusively related to the second bundle). Note further the five extremely negative emotions in the null class, which are not experienced towards any of the target person at any moment.

We now proceed with a discussion on how the three modes link together, that is, on the emotional evolution towards the respective target persons across time. Because five of the six target persons are in different classes that are almost not hierarchically related to each other, we will discuss this evolution for each of them separately, drawing parallels between them whenever needed.

We can read from the graph that the partner and Unstable relation A are completely stable relations (at least in the period under study) as the emotions experienced towards them do not vary at all across occasions. (This means that the characterization of unstability of Relation A is due either to past experiences before the onset of the study or to a prospect of possible unstability in the future.) 


\section{THREE-WAY HICLAS}

Furthermore, we can derive that the participant of the study experiences these two relations as entirely positive, as they elicit the full range of positive feelings over the complete five-month period.

With respect to her parents, we derive that also the relation with her father is entirely stable, although in addition to the full set of positive emotions, he elicits some (mildly) negative and ambivalent feelings as well. The analysis further shows that she has a slightly more negative relation with her mother as compared to her father given that her mother elicits the same emotions as her father, except the positive emotions of feeling a bond, admiration, respect, and feeling secure (during the entire period) and feeling easy (during the second phase [T7-T9]). The latter emotion is otherwise the only one that changes across time (feeling easy towards her mother at the beginning and the end, but not intermediate), so that the relation with her mother can be characterized as "almost stable".

The Unstable relations B and C are truly unstable, indeed. Note first that neither of these relations elicit strong positive feelings (such as feeling a bond, admiration, love, warmth, etc.) at any occasion. But they do elicit mild positive feelings (feeling at ease, comfortable, easy, etc.), although, for Relation B, this is only the case at the beginning (or first phase, T1-T6) and the end (or third phase, occasion T10) of the studied period, and, for Relation C, from the second phase onwards (phases two and three, occasions T7-T10). Both relations also elicit rather strong negative emotions (angry, close-hearted, etc.): For Relation B, this occurs at the end (during phases two and three) and for Relation $\mathrm{C}$ at the start and at the last occasion (during phases one and three). From these results, we may conclude that Relation B turned from positive to negative, while Relation C went from negative to positive and at the very last occasion back to negative.

It would definitely be interesting to confirm or disconfirm the conclusions drawn from this analysis in a discussion of the results with the participant. Unfortunately, no details are available about the target persons. Nevertheless, this study shows that a Tucker3-HICLAS analysis of longitudinal data from a single person may provide insightful results and interesting hypotheses about evolutions in this person.

\section{Concluding Remarks}

In this paper, we have discussed the INDCLAS and Tucker3-HICLAS models, which both are three-way extensions of De Boeck and Rosenberg's (1988) original hierarchical classes model. We have shown the similarities and differences between both models from a substantive rather than from a pure mathematical point of view. We further illustrated how a Tucker3-HICLAS analysis may improve on the results from an INDCLAS analysis. This improvement may manifest itself in two possible ways, which, to be sure, are aspects of the same underlying principle. First, a Tucker3-HICLAS model possibly implies a simplification of the hierarchical classifications of one or more modes as compared to an INDCLAS model, without the simplification (substantially) affecting the goodness of fit. This was exemplified in the hypothetical example in the first section of this paper as well as in the analysis of the situation-response data in

Application 1. Second, a Tucker3-HICLAS model of about the same complexity as an INDCLAS model (in terms of the number of parameters) may imply a sig- 


\section{APPLIED MULTIVARIATE RESEARCH}

nificant improvement in goodness of fit, which was illustrated in the application with interpersonal emotion data.

In the remainder of this paper, we want to draw the attention to some recent developments within the HICLAS family. In particular, we will discuss three that are especially interesting for practical users of INDCLAS and Tucker3-HICLAS as they may help users in obtaining robust models (i.e., models that can be expected to replicate across studies with a similar set up) and in providing sound substantive interpretations of these models. A first development relates to imposing restrictions on the model based on some a priori theory about the structure of the data, which is nowadays a common practice in factor analysis and is known as a confirmatory (as opposed to an exploratory) analysis. In the context of hierarchical classes analysis, Ceulemans et al. (2004) introduced various possible constraints and organized them in a taxonomy. They accordingly discussed adaptations to the existing algorithms so as to fit constrained models to observed data and provided tools so as to compare the goodness of fit of a constrained model to the corresponding unconstrained model. Such constraints are interesting both because of substantive reasons (a priori knowledge can be incorporated in the model) and for reasons of robustness of the fitted model (considering that in constrained models a smaller number of parameters has to be estimated). As an example, one may think of a confirmatory analysis using data similar to the hypothetical data in the first section of this paper, where (some of) the values of the symptom bundle matrix are fixed a priori based on a theory about which symptoms are characteristic of the hypothesized syndromes. Another example was already hinted at in the second application (on interpersonal emotions), in which one of the modes involved was a time mode and where we mentioned that an a priori constraint may be imposed so as to assure that the time classes are sets of consecutive moments.

When we discussed the hierarchical classification of the persons in the first application, we mentioned the possibility of relating external information to the obtained model and showed how traditional statistics (in this case, a polychoric correlation, but other statistics, e.g., based on analysis of variance) may be used to relate differences on a single covariate (in this case, sex) to differences in class membership. Sometimes, however, more than one covariate is available for the elements of a given mode, such that the external information may be organized in a two-way two-mode array (which then, as a consequence, has one mode in common with the data at hand). Suppose as an example that in the first application, in addition to the person by situation by response data, we have also acquired self-descriptions on a number of personality traits from each of the participants in the study; these external data can then be organized as a person by trait matrix (e.g., indicating whether or not the person assigns him-/herself the trait). For the case that this additional two-way two-mode array is binary, Wilderjans, Ceulemans, and Van Mechelen (2008) recently proposed a general framework, where the INDCLAS or Tucker3-HICLAS model for the person by situation by response data is estimated simultaneously with a two-way HICLAS model (such as De Boeck \& Rosenberg's, 1988, model) for the person by personality trait data, with the restriction that the person bundle matrix in the three-way HICLAS model is identical to the person bundle matrix in the two-way HICLAS model. The advantages of such a simultaneous approach are twofold: (a) more robust estima- 


\section{THREE-WAY HICLAS}

tion of the common bundle matrix (in this case, the person bundle matrix) is obtained and (b) the two submodels are integrated in an overall model from which the connection among the different modes are readily understood. In the example at hand, for instance, the overall model sheds light on how the structure of individual differences in behavioral signatures relates to individual differences in personality traits.

Finally, a third recent development comprises Leenen et al.'s (2008) Bayesian extension of hierarchical classes analysis, which, if considered for the models discussed in this paper, leads to Bayesian INDCLAS or Tucker3-HICLAS models. Such models would explicitly include a parameter for the probability that some random error process changes a predicted value of one to an observed value of zero or vice versa, and, as such, account for the discrepancies between predictions $(\underline{\mathbf{X}})$ and data $(\underline{\mathbf{X}})$. Estimation techniques that are common in Bayesian analysis, such as Markov Chain Monte Carlo algorithms, are then employed to fit the model to the data. The most important advantages of the Bayesian approach include: (a) Rather than pinning the user down to just a single best-fitting model solution for a given data array (as the traditional INDCLAS and Tucker3HICLAS algorithms do), the Bayesian algorithm aims at returning all good model solutions (with "good" refering to goodness of fit to the data). Subsequently, common patterns across this set of good models may be looked for, as such separating robust from incidental results. (b) Statistically sound tools for model selection (particularly, rank selection) and model testing become available; these tools allow for the testing of very specific model assumptions with tests driven by substantive considerations.

\section{REFERENCES}

Akaike, H. (1973). Information theory and an extension of the maximum likelihood principle. In B. N. Petrov \& F. Csaki (Eds.), Second international symposium on information theory (pp. 267-281). Budapest, Hungary: Academiai Kiado.

Bozdogan, H. (1987). Model selection and Akaike's Information Criterion (AIC): The general theory and its analytical extensions. Psychometrika, 52, 345370.

Cattell, R. B. (1966). The meaning and strategic use of factor analysis. In R. B. Cattell (Ed.), Handbook of multivariate experimental psychology (pp. 174243). Chicago: Rand McNally.

Ceulemans, E., \& Van Mechelen, I. (2003). Uniqueness of $N$-way $N$-mode hierarchical classes models. Journal of Mathematical Psychology, 47, 259-264.

Ceulemans, E., \& Van Mechelen, I. (2004). Tucker2 hierarchical classes analysis. Psychometrika, 69, 375-399.

Ceulemans, E., \& Van Mechelen, I. (2005). Hierarchical classes models for threeway three-mode binary data: Interrelations and model selection. Psychometrika, 70, 461-480.

Ceulemans, E., Van Mechelen, I., \& Kuppens, P. (2004). Adapting the formal to the substantive: Constrained Tucker3-HICLAS. Journal of Classification, $21,19-50$. 


\section{APPLIED MULTIVARIATE RESEARCH}

Ceulemans, E., Van Mechelen, I., \& Leenen, I. (2003). Tucker3 hierarchical classes analysis. Psychometrika, 68, 413-433.

Coombs, C. H. (1964). A theory of data. New York: Wiley.

Davey, B. A., \& Priestley, H. A. (2002). Introduction to lattices and order (Second ed.). Cambridge, UK: University Press.

De Boeck, P., \& Rosenberg, S. (1988). Hierarchical classes: Model and data analysis. Psychometrika, 53, 361-381.

Doignon, J.-P., \& Falmagne, J.-C. (1999). Knowledge spaces. Berlin, Germany: Springer.

Falmagne, J.-C., Koppen, M., Villano, M., Doignon, J.-P., \& Johannesen, L. (1990). Introduction to knowledge spaces: How to build, test and search them. Psychological Review, 97, 201-224.

Gara, M. A. (1990). A set-theoretic model of person perception. Multivariate Behavioral Research, 25, 275-293.

Gara, M. A., \& Rosenberg, S. (1979). The identification of persons as supersets and subsets in free-response personality descriptions. Journal of Personality and Social Psychology, 37, 2161-2170.

Gati, I., \& Tversky, A. (1982). Representations of qualitative and quantitative dimensions. Journal of Experimental Psychology: Human Perception and Performance, 8, 325-340.

Geluykens, K. (2000). Scripts over emoties binnen relaties [Emotion scripts within relations]. Unpublished master's thesis, Katholieke Universiteit Leuven.

Kiers, H. A. L. (2000). Towards a standardized notation and terminology in multiway analysis. Journal of Chemometrics, 14, 105-122.

Kuppens, P., Van Mechelen, I., Smits, D. J. M., De Boeck, P., \& Ceulemans, E. (2007). Individual differences in patterns of appraisal and anger experience. Cognition \& Emotion, 21, 689-713.

Leenen, I., \& Van Mechelen, I. (2001). An evaluation of two algorithms for hierarchical classes analysis. Journal of Classification, 18, 57-80.

Leenen, I., Van Mechelen, I., \& De Boeck, P. (1999). A generic disjunctive/ conjunctive decomposition model for $n$-ary relations. Journal of Mathematical Psychology, 43, 102-122.

Leenen, I., Van Mechelen, I., De Boeck, P., \& Rosenberg, S. (1999). INDCLAS: A three-way hierarchical classes model. Psychometrika, 64, 9-24.

Leenen, I., Van Mechelen, I., Gelman, A., \& De Knop, S. (2008). Bayesian hierarchical classes analysis. Psychometrika, 73, 39-64.

Mischel, W., \& Shoda, Y. (1995). A cognitive-affective system theory of personality: Reconceptualizing situations, dispositions, dynamics, and invariance in personality structure. Psychological Review, 102, 246-268.

Schepers, J., \& Van Mechelen, I. (2006). The real-valued model of hierarchical classes. Manuscript submitted for publication.

Shoda, Y., Mischel, W., \& Wright, J. C. (1994). Intraindividual stability in the organization and patterning of behavior: Incorporating psychological situations into the idiographic analysis of personality. Journal of Personality and Social Psychology, 67, 674-687.

Tucker, L. R. (1964). The extension of factor analysis to three-dimensional matrices. In N. Frederiksen \& H. Gulliksen (Eds.), Contributions to mathematical psychology (pp. 109-127). New York: Holt, Rinehart, \& Winston. 


\section{THREE-WAY HICLAS}

Van Mechelen, I., \& De Boeck, P. (1989). Implicit taxonomy in psychiatric diagnosis: A case study. Journal of Social and Clinical Psychology, 8, 276287.

Van Mechelen, I., De Boeck, P., \& Rosenberg, S. (1995). The conjunctive model of hierarchical classes. Psychometrika, 60, 505-521.

Van Mechelen, I., Lombardi, L., \& Ceulemans, E. (2007). Hierarchical classes modeling of rating data. Psychometrika, 72, 475-488.

Van Mechelen, I., Rosenberg, S., \& De Boeck, P. (1997). On hierarchies and hierarchical classes models. In B. Mirkin, F. R. McMorris, F. S. Roberts, \& A. Rzhetsky (Eds.), Mathematical hierarchies and biology (pp. 291-298). Providence, RI: American Mathematical Society.

Van Mechelen, I., \& Van Damme, G. (1994). A latent criteria model for choice data. Acta Psychologica, 87, 85-94.

Vansteelandt, K., \& Van Mechelen, I. (1998). Individual differences in situationbehavior profiles: A triple typology model. Journal of Personality and Social Psychology, 75, 751-765.

Wilderjans, T., Ceulemans, E., \& Van Mechelen, I. (2008). The CHIC model: A global model for coupled binary data. Psychometrika, 73, 729-751.

Author Note

Iwin Leenen is now at the Instituto Mexicano de Investigación de Familia y Población, Mexico; Eva Ceulemans is at the Department of Educational Sciences, K.U.Leuven.

At the time of writing, Iwin Leenen was a post-doctoral fellow of the Ministerio de Educación y Ciencia, Spain, and Eva Ceulemans was a post-doctoral fellow of the Fund for Scientific Research, Flanders (Belgium). The research reported in this paper was partially supported by the Research Council of K.U.Leuven (GOA/05/04). The authors wish to thank Pablo Gaviño Castellanos for his careful reading and suggestions on a previous version of this manuscript.

Correspondence concerning this article should be addressed to Eva Ceulemans, Department of Educational Sciences, K.U.Leuven, Andreas Vesaliusstraat 2 - PO Box 3762, B-3000 Leuven, Belgium. E-mail: Eva.Ceulemans@ped. kuleuven.be.

\section{Footnotes}

1. We adhere to the notation proposed by Kiers (2000) in representing threedimensional arrays by underlined bold uppercase symbols (while for matrices, the standard, nonunderlined bold uppercase symbol is used).

2. As we will explain in the next section, a model may be obtained by applying the INDCLAS or Tucker3-HICLAS algorithm to the three-way three-mode data. The Tucker3-HICLAS algorithm (which include the INDCLAS algorithm as a special case) is freely available from http://ppw.kuleuven.be/okp/t3h (upon filling out a form, you will be sent a link to a ZIP file with the program and some example data sets). The reader who is interested in the intricacies of the algorithm is referred to the original papers (Ceulemans et al., 2003; Leenen, Van Mechelen, De Boeck, \& Rosenberg, 1999). 


\section{APPLIED MULTIVARIATE RESEARCH}

3. Note that the mode of which the elements/classes appear in the circles is somehow selected arbitrarily. In most cases, the user will opt for the mode for which the hierarchical organization of its elements is of least interest.

4. The reader may wonder why we do not simply calculate the respective angles by standard geometry formulae. In this respect, note that the order among the angles is not invariant under linear transformations of the badness-of-fit statistic used (e.g., different results may be obtained from a plot with number of discrepancies instead of proportion of discrepancies on the ordinate). The scree tests proposed by Ceulemans and colleagues overcome this drawback. 